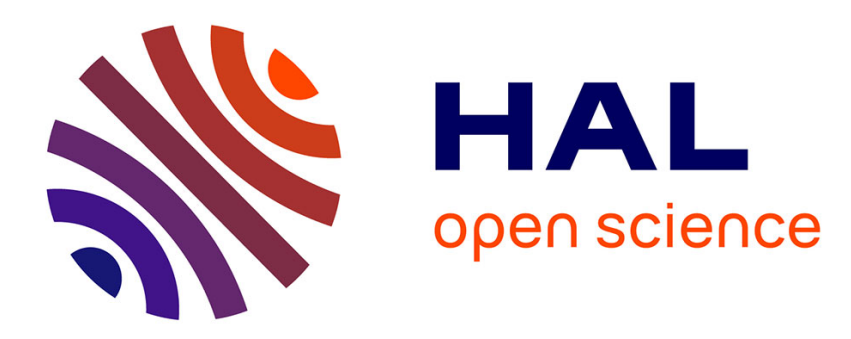

\title{
Computer Science Must Rely on Strongly-Typed Actors and Theories for Cybersecurity \\ Carl Hewitt
}

\section{To cite this version:}

Carl Hewitt. Computer Science Must Rely on Strongly-Typed Actors and Theories for Cybersecurity.

Inference Robustness, In press. hal-02165430v2

\section{HAL Id: hal-02165430 \\ https://hal.science/hal-02165430v2}

Submitted on 10 Jul 2019

HAL is a multi-disciplinary open access archive for the deposit and dissemination of scientific research documents, whether they are published or not. The documents may come from teaching and research institutions in France or abroad, or from public or private research centers.
L'archive ouverte pluridisciplinaire HAL, est destinée au dépôt et à la diffusion de documents scientifiques de niveau recherche, publiés ou non, émanant des établissements d'enseignement et de recherche français ou étrangers, des laboratoires publics ou privés. 


\section{HAL Open Archives. https://hal.archives-ouvertes.fr \\ Computer Science Must Rely on Strongly-Typed Actors and Theories for Cybersecurity \\ Carl Hewitt ${ }^{\dagger}$}

This article shows how fundamental higher-order theories of mathematical structures of computer science (e.g. natural numbers [Dedekind 1888] and Actors [Hewitt et. al. 1973]) are categorical meaning that they can be axiomatized up to a unique isomorphism thereby removing any ambiguity in the mathematical structures being axiomatized. Having these mathematical structures precisely defined can make systems more secure because there are fewer ambiguities and holes for cyberattackers to exploit. For example, there are no infinite elements in models for natural numbers to be exploited. On the other hand, the $1^{\text {st }}$-order theories and computational systems which are not strongly-typed necessarily provide opportunities for cyberattack.

Cyberattackers have severely damaged national, corporate, and individual security as well causing hundreds of billions of dollars of economic damage. [Sobers 2019] A significant cause of the damage is that current engineering practices are not sufficiently grounded in theoretical principles. In the last two decades, little new theoretical work has been done that practically impacts large engineering projects with the result that computer systems engineering education is insufficient in providing theoretical grounding. If the current cybersecurity situation is not quickly remedied, it will soon become much worse because of the projected development of Scalable Intelligent Systems by 2025 [Hewitt 2019].

Kurt Gödel strongly advocated that the Turing Machine is the preeminent universal model of computation. A Turing machine formalizes an algorithm in which computation proceeds without external interaction. However, computing is now highly interactive, which this article proves is beyond the capability of a Turing Machine. Instead of the Turing Machine model, this article presents an axiomatization of a strongly-typed universal model of digital computation (including implementation of Scalable Intelligent Systems [Hewitt 2019]) up to a unique isomorphism. Strongly-typed Actors provide foundations for tremendous improvements in cyberdefense.

Index Terms - uniquely categorical theories, strong types, Scalable Intelligent Systems, Actor Model of Computation, Gul Agha, Joe Armstrong, Sergei Artemov, Russ Atkinson, Jeremy Avigad, Steve Awodey, Henry Baker, Jon Barwise, Nicolas Bourbaki, Cesare Burali-Forti, John Burgess, Will Clinger, Per Brinch Hansen, Alonzo Church, Thierry Coquand, Haskell Curry, Ole-Johan Dahl, Richard Dedekind, Jean-Yves Girard, Kurt Gödel, Irene Greif, John Harrison, Tony Hoare, Gérard Huet, Ken Kahn, Richard Karp, Bill Kornfeld, Thomas Kuhn, Imre Lakatos, Martin Löb, Per Martin-Löf, Penelope Maddy, John McCarthy, Robin Milner, Kristen Nygaard, Gordon Plotkin, Bertrand Russell, Alan Turing, Ludwig Wittgenstein, John Woods, Stephen Yablo, Akinori Yonezawa

\section{INTRODUCTION}

The approach in this article is to embrace all of the most powerful tools of classical mathematics in order to provide mathematical foundations for Computer Science. Fortunately, the results presented in this article are technically simple so they can be readily automated, which will enable better collaboration

${ }^{\dagger}$ C. Hewitt is the Board Chair of iRobust (International Society for Inconsistency Robustness) and an emeritus professor of MIT. His homepage is https://professorhewitt.blogspot.com/ 
between humans and computer systems.

Mathematics in this article means the precise formulation of standard mathematical theories that axiomatize the following standard mathematical structures up to a unique isomorphism: Booleans, natural numbers, reals, ordinals, set of elements of a type, computable procedures, and Actors, as well as the types and theories of these structures.

In a strongly typed mathematical theory, every proposition, mathematical term, and program expression has a type. Types are constructed bottom up from mathematical types that are individually categorically axiomatized in addition to the types of a theory being categorically axiomatized as a whole.

[Russell 1906] introduced types into mathematical theories to block paradoxes such as The Liar which could be constructed as a paradoxical fixed point using the mapping $\mathrm{p} \mapsto \neg \mathrm{p}$ (notation from [Bourbaki 1939-2016]), except for the requirement that each proposition must have an order beginning with $1^{\text {st }}$-order. Since $\mathrm{p}$ is a propositional variable in the mapping, $\neg \mathrm{p}$ has order one greater than the order of $\mathrm{p}$. Thus because of orders on propositions, there is no paradoxical fixed point for the mapping $\mathrm{p} \mapsto \neg \mathrm{p}$ which if it existed could be called I'mFalse such that I'mFalse $\Leftrightarrow \neg I$ I'mFalse. Unfortunately in addition to attaching orders to propositions, Russell also attached orders to the other mathematical objects (such as natural numbers), which made the system unsuitable for standard mathematical practice.

\section{LIMITATIONS OF $1^{\text {ST }}$-ORDER LOGIC FOUNDATIONS}

Wittgenstein correctly proved that allowing the proposition I'mUnprovable [Gödel 1931] into Russell's foundations for mathematics infers a contradiction as follows:

"Let us suppose [Gödel 1931 was correct and therefore] I prove the unprovability (in Russell's system) of [Gödel's I'mUnprovable] $P$; [i.e., $\vdash_{\mathbb{R} \text { urssielll }} \nvdash_{\mathbb{R} \text { iussiellll }} P$ where $P \Leftrightarrow \nvdash_{\mathbb{R} \text { iussielll }} P$ ] then by this proof I have proved $P$ [i.e., $\vdash_{\mathbb{R} u r s s e l l l} P$ because $P \Leftrightarrow \nvdash_{\mathbb{R} \text { urssellll }} P$ ]. Now if this proof were one in Russell's system [i.e., $\left.\vdash_{\mathbb{R} u r s s i l l l} \vdash_{\mathbb{R} \text { iussielll }} P\right]$ - I should in this case have proved at once that it

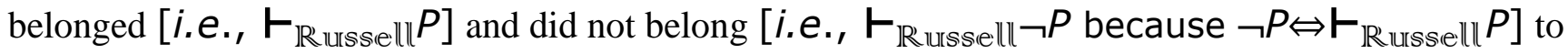
Russell's system. But there is a contradiction here! [i.e., $\vdash_{\mathbb{R} \text { urssiellll }} P$ and $\vdash_{\mathbb{R} \text { irssielll }} \neg P$ ] ...

[This] is what comes of making up such propositions." [emphasis added] [Wittgenstein 1978]

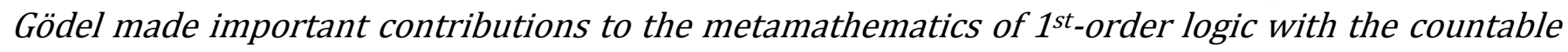
compactness theorem and formalization of provability. [Gödel 1930] However decades later, Gödel asserted that the [Gödel 1931] inferential undecidability results were for a $1^{\text {st }}$-order theory instead of the theory $\mathbb{R}$ urssellll, which is an extension of Russell's theory by adding the natural numbers induction axiom as stated in [Gödel 1931]. In this way, Gödel dodged the point of Wittgenstein's criticism.

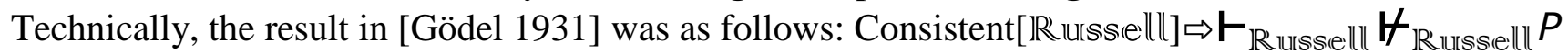
where $P \Leftrightarrow \forall_{\mathbb{R} u r s s e l l l l} P$ and Consistent[Rurssellll] if an only if there is no proposition $\Psi$ such that $\vdash_{\mathbb{R} \text { urssielll }} \Psi \wedge \neg \Psi$, However, Wittgenstein was understandably taking it as a given that $\mathbb{R}$ urssielll is consistent because it formalized standard mathematical practice and had been designed to block known paradoxes (such as The Liar) using orders on propositions. Consequently, Wittgenstein elided the result in [Gödel 1931] to $\vdash_{\mathbb{R} \text { irssellll}} \forall_{\mathbb{R} \text { iussellll}} P$. His point was that Rursselll is consistent provided that the proposition

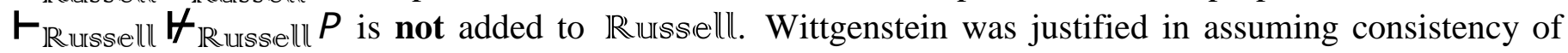
Riussielll because the standard theory of natural numbers is arguably consistent because it has a model. [Dedekind 1888] See [Shanker 1988] for further discussion of Wittgenstein on Gödel's results.

According to [Russell 1950]: "A new set of puzzles has resulted from the work of Gödel, especially his article [Gödel 1931], in which he proved that in any formal system [with recursively enumerable theorems] it is possible to construct sentences of which the truth [i.e., provability] or falsehood [i.e., unprovability] cannot be decided within the system. Here again we are faced with the essential 
necessity of a hierarchy [of sentences], extending upwards ad infinitum, and logically incapable of completion." [Urquhart 2016] Construction of Gödel's I'mUnprovable is blocked because the mapping $\Psi \mapsto \not \forall \Psi$ does not have a fixed point because the order of $\forall \Psi$ is one greater than the order of $\Psi$ since $\Psi$ is a propositional variable.

Although $1^{\text {st }}$-order propositions can be useful (e.g. in $1^{\text {st }}$-order proposition satisfiability testers), $1^{\text {st }}$-order theories are unsuitable as the mathematical foundation of computer science for the following reasons:

- Compactness Every $1^{\text {st }}$-order theory is compact [Gödel 1930] (meaning that every countable inconsistent set of propositions has a finite inconsistent subset). Compactness is false of the standard theory of natural numbers for the following reason: if $\mathrm{k}$ is a natural number then the set of propositions of the form $\mathrm{i}>\mathrm{k}$ where $\mathrm{i}$ is a natural number is inconsistent but has no finite inconsistent subset, thereby contradicting compactness.

- Monsters Every $1^{\text {st }}$-order theory is ambiguous about fundamental mathematical structures such as the natural numbers, lambda expressions, and Actors [Hewitt and Woods assisted by Spurr 2019]. For example,

o Every $1^{\text {st }}$-order axiomatization of the natural numbers has a model with an element (which can be called $\infty$ ) for a natural number, which is a "monster" [Lakatos 1976] because $\infty$ is larger than every standard natural number.

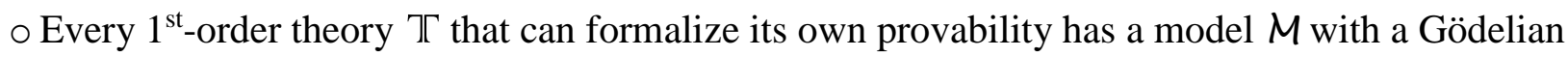

"monster" element proposition $\Gamma$ that proves $\rceil \Gamma$ inconsistent (i.e. $\vDash_{M} \vdash_{\rceil} \Gamma \wedge \neg \Gamma$ ) by the following proof:

According to [Gödel 1931], $\forall_{\rrbracket}$ Consistent[ТГ] and consequently because of the $1^{\text {st }}$-order model

"completeness" theorem [Gödel 1930] there must be some model $M$ of $Т$ in which Consistent[Т] is false. [cf. Artemov 2019]

Such monsters are highly undesirable in models of standard mathematical structures in Computer Science because they are inimical to model use.

- Inconsistency This article shows that a theory with recursively enumerable theorems that can formalize its own provability is inconsistent.

- Intelligent Systems. If a $1^{\text {st }}$-order theory is not consistent, then it is useless because each and every proposition (no matter how nonsensical) can be proved in the theory. However, Scalable Intelligent Systems must reason about massive amounts of pervasively-inconsistent information. [Hewitt and Woods assisted by Spurr 2019] Consequently, such systems cannot always use $1^{\text {st }}$-order theories. Conversational Logic [Hewitt 2016-2019] needs to be used to reason about inconsistent information in Scalable Intelligent Systems. [cf. Woods 2013]

Consequently, Computer Science must move beyond $1^{\text {st }}$-order logic for its foundations.

\section{STRONG TYPES}

Types must be strong to prevent inconsistency but flexible to allow all valid inference. (See appendix on how known paradoxes are blocked.) Although mathematics in this article necessarily goes beyond $1^{\text {st }}$-order logic, standard mathematical practice is used. Wherever possible, previously used notation is employed. The following notation is used for types:

- The notation $x: t$ means that $\mathrm{x}$ is of type $t$. For example, $0: N$ expresses that 0 is of type $N$, which is the type of a natural number. Types are intensional, i.e., if $\mathrm{x}: t_{1} \Leftrightarrow \mathrm{x}: t_{2}$ for every $\mathrm{x}$ does not mean that $t_{1}=t_{2}$ where $t_{1}$ and $t_{2}$. are types. Burali-Forti/Girard paradox is blocked because for every type $t, \neg t$. $t$ and is $t$ is of type Typeof $\triangleleft t \triangleright$. 
- $t_{2}{ }^{t 1}$ is type of all functions from $t_{1}$ into $t_{2}$ where $t_{1}$ and $t_{2}$. are types. A function is total and may be uncomputable. For example, $N^{N}$ is the type all total functions from natural numbers into the natural numbers, which are uncountable. If $\mathrm{f}: N^{N}$, then $\mathrm{f}[3]$ is the value of function $\mathrm{f}$ on argument 3.

- $t_{1} \rightarrow t_{2}$ is type of nondeterministic computable procedures from $t_{1}$ into $t_{1}$ where $t_{1}$ and $t_{2}$ are types whereas $t_{1} \rightarrow_{1} t_{2}$ is the deterministic procedures. For example, [] $\rightarrow$ Boolean is the type all partial nondeterministic procedures of no argument into the type of Boolean. If $\mathrm{p}:[] \rightarrow$ Boolean, then $\mathrm{p}$.[ ] starts a computation by providing input [ ] to procedure $\mathrm{p}$ which might return True or return False. It also might happen that $\mathrm{p}-[]$ does not return a value.

- $\left[t_{1}, t_{2}\right]$ is type of pairs of $t_{1}$ and $t_{2}$ where $t_{1}$ and $t_{2}$ are types. For example, [N, Boolean] is the type of pairs whose first is a natural number and whose second is a Boolean.

- Propositionoforder $\triangleleft i \triangleright$ is type of a proposition of order i where $i: N_{+}$and $N_{+}$is the type of positive natural numbers. For example, Propositionoforder $\triangleleft 1 \triangleright$ is the type of propositions of order 1.

$\circ$ Proposition $\Psi$ means $\exists\left[\mathrm{i}: N_{+}\right] \Psi:$ Propositionoforder $\triangleleft \mathrm{i} \triangleright$

- P predicateOn $t$ means $\exists\left[\mathrm{i}: N_{+}\right]$P:Propositionoforder $\triangleleft \mathrm{i} \triangleright{ }^{t}$

- $t \underline{\exists} \mathrm{P}$ is the type of $t$ restricted to $\mathrm{P}$ where $t$ is a type and $\mathrm{P}$ is a predicate. For example, replacement for types is expressed using restriction, i.e., the range of a function $\mathrm{f}: t_{2}^{t_{1}}$ is $t_{2} \exists \mathrm{y} \mapsto \exists\left[\mathrm{x}: t_{1}\right] \mathrm{y}=\mathrm{f}[\mathrm{x}]$.

- Typeof $\triangleleft t \triangleright$ is the type of the type $t$. For example, $N$ :Typeof $\triangleleft N \triangleright$ (cf. [Martin-Löf. 1998])

Types are constructed bottom-up from types that are categorically axiomatized up to a unique isomorphism. Type checking is linear in the size of the propositon, mathematical term or procedural expression to be type checked. See appendix for syntax of propositions, mathematical terms, and procedural expressions.

\section{FOUNDATIONAL THEORY OF DigITAL COMPUTATION}

Cybersecurity requires that fundamental mathematical structures in Computer Science must be precisely defined. This section shows how to axiomatize classical nondeterministic computable procedures up to a unique isomorphism including computable nondeterministic procedures that cannot be implemented using a Lambda Expression [Church 1932] or Turing Machine [Turing 1936].

Computation that cannot be done by $\lambda$ Calculus, Nondeterministic Turing Machines, or pure Logic Programs

[Church 1931] and [Turing 1936] developed equivalent models of computation based on the concept of an algorithm, which by definition is provided an input from which it is to compute a value without external interaction. After physical computers were constructed, they soon diverged from computing only algorithms meaning that the Church/Turing theory of computation no longer applied to computation in practice because computer systems are highly interactive as they compute, which inspired the development of the Actor Model in 1972 to characterize all digital computation.

Theorem. An Actor machine can perform computations that a no $\lambda$ expression, nondeterministic Turing

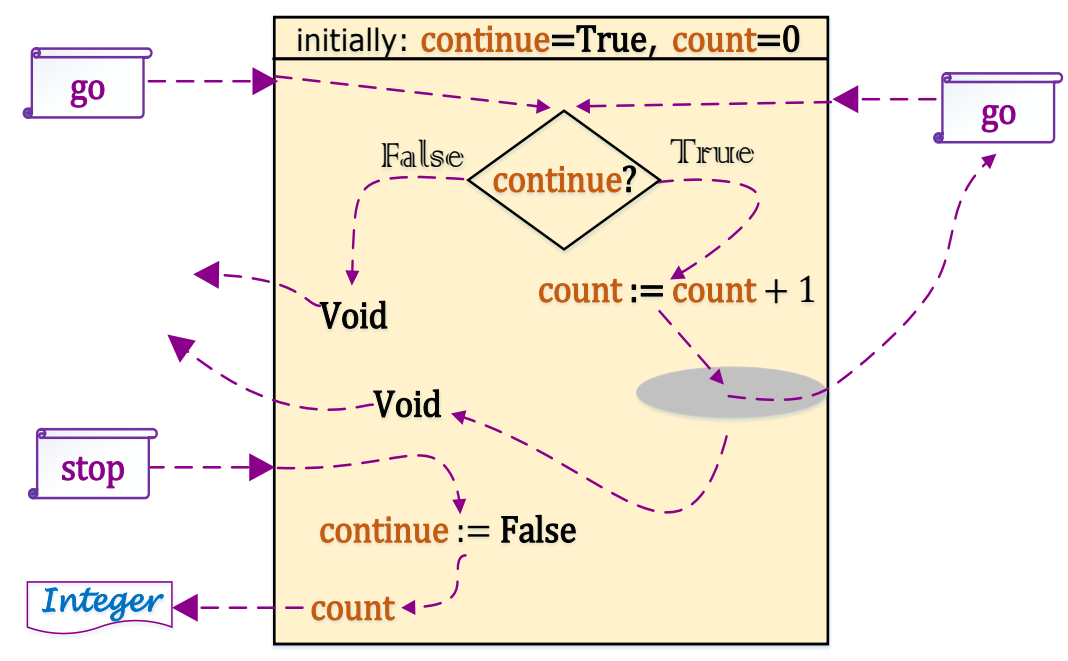

Resend go message until stop message received 
Machine or pure Logic Program can implement because there is an always-halting Actor machine that can compute an integer of unbounded size (cf. [Clinger 1981]) This can be accomplished using an Actor with a variable count that is initially 0 and a variable continue initially True. The computation is begun by concurrently sending two messages to the Actor machine: a stop request that will return an integer and a go

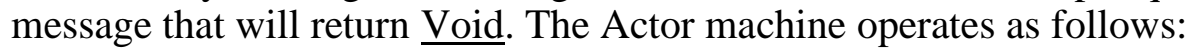

- When a stop message is received, return count and set continue to False for the next message received.

- When a go message is received:

- If continue is True, increment count by 1 , send this Actor machine a go message in a hole of the region of mutual exclusion, and afterward return $\underline{\text { Void. }}$.

o If continue is False, return Void.

Theorem. There is no $\lambda$ expression, nondeterministic Turing Machine, Parallel Program Schemata [Karp and Miller 1967] or pure Logic Program [Hewitt 1969] that implements the above computation.

Proof [Plotkin 1976]:

"Now the set of initial segments of execution sequences of a given nondeterministic program $\mathrm{P}$, starting from a given state, will form a tree. The branching points will correspond to the choice points in the program. Since there are always only finitely many alternatives at each choice point, the branching factor of the tree is always finite. That is, the tree is finitary. Now König's lemma says that if every branch of a finitary tree is finite, then so is the tree itself. In the

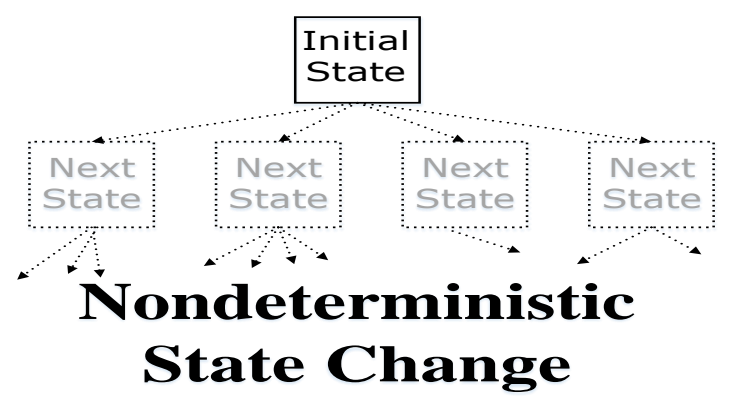
present case this means that if every execution sequence of $\mathrm{P}$ terminates, then there are only finitely many execution sequences. So if an output set of $\mathrm{P}$ is infinite, it must contain a nonterminating computation."

A classification should be developed for nondeterministic computable procedures that are not Church/Turing computable, e.g., in terms of messages sent.

An Actor machine can be thousands of times faster than any corresponding pure Logic Program or parallel nondeterministic $\lambda$ expression. (cf. [Kahn 1979, Kornfeld 1981, Hewitt and Woods assisted by Spurr 2019]). Since the time of this early work, Actors have grown to be one of the most important paradigms in computing [Hewitt and Woods assisted by Spurr 2019, Hoare 2018, Milner 1993].

Limitations of $1^{\text {st }}$-order Logic for Concurrent Computation

Theorem. It is well known that there is no $1^{\text {st }}$-order theory for the above Actor machine.

Proof. Every $1^{\text {st }}$-order theory is compact meaning that every inconsistent set of propositions has a finite inconsistent subset. Consequently, to show that there is no $1^{\text {st }}$-order theory, it is sufficient to show that there is an inconsistent set of propositions such that every finite subset is consistent. Let Output[i] mean that $\mathrm{i}$ is output. Then the set of propositions $\exists[\mathrm{i}: \mathrm{N}] \neg$ Output[i] is inconsistent but every finite subset $S$ is consistent because the Actor machine output might be larger than any output in $S$.

Interactive computation has fundamentally transformed the foundations and practice of computation since the initial non-interactive conceptions of [Church 1931] and [Turing 1936]. Although $1^{\text {st }}$-order propositions can be useful (e.g. in testing $1^{\text {st }}$-order propositions for satisfiability), interactive concurrency in Actor systems illustrate why $1^{\text {st }}$-order logic cannot be the foundation for theories in Computer Science.

Actors in Practice

An interface can be defined using an interface name, "interface", and a list of message handler signatures, where message handler signature consists of a message name followed by argument types delimited by "[" and "]", " $\rightarrow$ ", and a return type. For example, the interface type ReadersWriter can be defined as follows:

Readerswriter interface $\operatorname{read}[Q$ uery] $\rightarrow$ ReadResponse, // results of read query on database write[Update] $\rightarrow$ WriteResponse // perform update on database 
Below is an implementation of Readerswriter that does not allow concurrency in aDatabase: OneAtATime [aDatabase:ReadersWriter] implements Readerswriter $\operatorname{read}[$ aQuery] $\mapsto \quad / /$ read[aQuery] message received

aDatabase.read[aQuery] // forward query while not receiving any messages until finished write[anUpdate] $\mapsto$ // write[anUpdate] request received

aDatabase.read[aQuery] // forward request while not receiving any messages until finished Although, the OneAtATime implementation of Readerswriter excludes all other activity when there is writing activity in aDatabase, it does not allow multiple reading activities when there is no writing activity.

\section{Holes in regions of mutual exclusion}

In order to implement more general scheduling policies, a region of mutual exclusion can have holes (cf. [Atkinson 1980, Brinch Hansen 1996, Hewitt and Atkinson 1979, Hoare 1974]).

Below is an implementation SchedulerManger with facets writePriority, and readPriority that both implement Readerswriter:

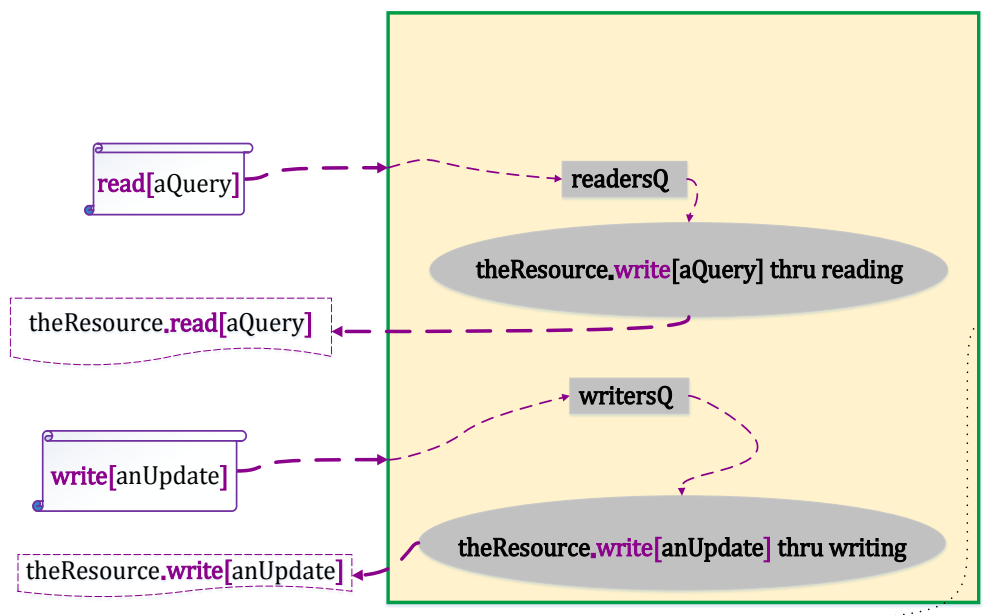

\section{SchedulerManger [aDatabase:Readerswriter] actor implements Scheduler}

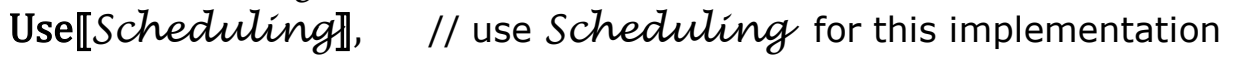

Contained【 // contained locals cannot be sent to other Actors

writersQ $\leftarrow$ New Suspended [], // FIFO of suspended writers

readersQ $\leftarrow$ New Suspended [], // FIFO of suspended readers reading $\leftarrow$ New Pending [], // set of active readers

writing $\leftarrow$ New Pending [1]], // at most 1 member in set of active writers

$/ /$ Invariant: Nonempty[writing] $\Rightarrow$ IsEmpty[reading]

getWritePriority $\mapsto \underline{\text { As }}$ writePriority, $\quad / /$ get writePriority facet

getReadPriority $\mapsto \underline{\text { As }}$ readPriority, $\quad / /$ get readPriority facet

upgrade[newVersion] $\mapsto / *$ upgrade request received $* /\{$ // upgrade in place to a new version

\section{CancellAll Scheduting \\ // CancellAll implementation from Scheduling}

(readersQ, writersQ, reading, writing);

// Require: AllEmpty[readersQ, writersQ, reading, writing]

Become newVersion\}

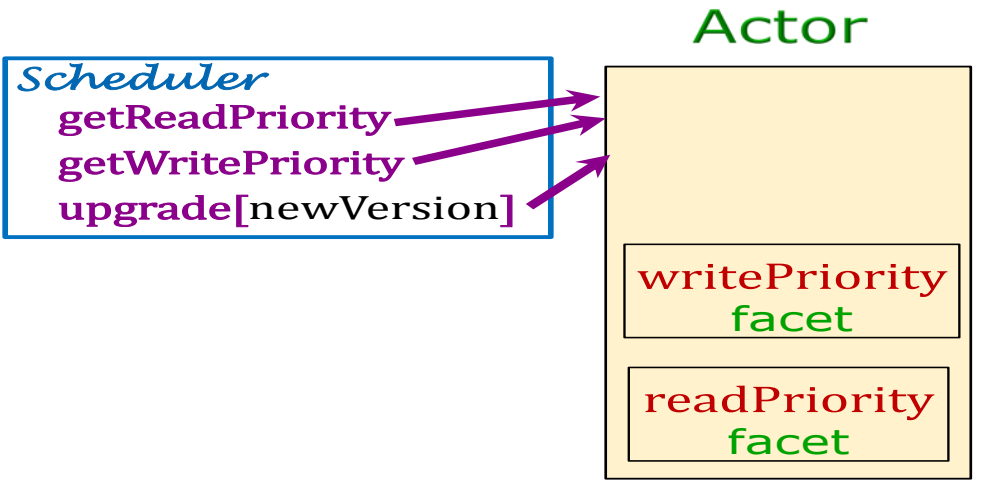


Different scheduling policies can be implemented by facets of SchedulerManger (cf. [Amborn 2004, Crahen 2002]) as shown below in this section.

A write priority policy can provide readers with more recent information (but with potentially less throughput) than a read priority policy. Below is an implementation of the writePriority facet of SchedulerManager:

writePriority facet SchedulerManager implements Readerswriter $\operatorname{read}[$ aQuery] $\mapsto / *$ read query received $* /\{$

Enqueue readers $Q$ when SomeNonempty(writing, writersQ readersQ);

// Require: IsEmpty[writing]

aDatabase read[aQuery] thru reading // forward query while recording pending in reading afterward // Require: IsEmpty[writing]

permit writersQ when IsEmpty(reading) else (readersQ when IsEmpty(writersQ))\},

Below is an implementation of doWrite which is an implementation of SchedulerManager that has been separated out for convenient reuse in different facets:

doWrite implementation SchedulerManger

write`Readers Writer [anUpdate] $\mapsto / *$ write request of ReadersWriter received */ \{

Enqueue writersQ when SomeNonempty(reading, writing, readersQ, writersQ);

// Require: AllEmpty[writing, reading]

aDatabase.write[anUpdate] thru writing // forward request while recording pending in writing afterward // Require: AllEmpty[writing, reading]

permit readersQ else writersQ\}

A read priority policy can have more throughput (but readers can potentially get less recent information) than a write priority policy. Below is an implementation of the readPriority facet of SchedulerManager:

readPriority facet SchedulerManager implements ReadersWriter

read[aQuery] $\mapsto / *$ read query received $* /\{$

Enqueue readers $Q$ when SomeNonempty(writing, writers $Q$ readers $Q)$;

// Require: IsEmpty[writing]

aDatabase.read[aQuery] thru reading // forward query while recording pending in reading permit readersQ // implements read priority policy

afterward // Require: IsEmpty[writing]

permit writersQ when IsEmpty(reading) else (readersQ when IsEmpty(writersQ))】\},

Note:

write`doWrite // write method from doWrite implementation of SchedulerManager

1. At most one activity is allowed to execute in the region of mutual exclusion of an Actor.

2. The region of mutual of exclusion has holes illustrating that an Actor is not a sequential process (thread) in which control moves sequentially through a program. Instead control moves through an Actor in accord with the scheduling performed by the Actor in response to communications received.

3. An implementation, e.g. SchedulerManager, differs from a class [Dahl and Nygaard 1967] as follows:

- An implementation can use multiple other implementations (thereby avoiding having to copy and paste code) using qualified names to prevent ambiguity, i.e., not relying on default selections in ambiguous cases as in C++ [ISO 2017].

- An implementation cannot be subclassed in order to prevent impersonation by other types. 
4. An invariant for an Actor must hold when it is created and when entering/leaving a continuous section of a region of mutual exclusion.

5. Strong types are the foundation of Actor communication. For example, if $\mathrm{x}$ is of type

ReadPriority, then x.getSchedular means ReadPriority.send[getSchedular to $\mathrm{x}$ ]

Types manage crypto without requiring programming by application programmers.

Theorem. Readers exclude writers from a database. Suppose manager 1 is

New ReadPriority[database 1 ]. After manger 1 has sent a write request to database 1 , it will not send another request to until it has received a response because the invariant

Nonempty[writing ] $\Rightarrow$ IsEmpty[reading ] holds as follows:

- The invariant holds when a ReadPriority implementation is created.

- If the invariant holds in a ReadPriority implementation when a communication is received, then it holds when has been processed.

Theorem. New ReadPriority【database $1 \rrbracket$ forwards messages to database 1 . Starvation of activities suspended in readers $Q$ and writers $Q$ as is prevented in a ReadPriorityimplementation as follows:

- An activity in readersQ progresses when

1. A read to the database is started by another activity

2. If writersQ and writing are both empty after the read to the database is completed by another activity

3. Else after the next write to the database is finished.

- An activity in writersQ progresses when

1. If readers $Q$ is empty when a write to the database is completed by another activity

2. Else when reading becomes smaller when reading the database is completed by another activity.

Reading throughput is maintained by permitting readersQ when another activity starts a read to the database.

\section{Axiomatization of Actors up to a unique isomorphism}

Let $\mathrm{x}[\mathrm{e}]$ be the behavior of Actor $\mathrm{x}$ at local event $\mathrm{e}$, Com be the type for a communication, and Behavior be the type for a procedure that maps a communication received to an outcome that has a finite set of created Actors, a finite set of sent communications, and a behavior for the next communication received.

The theory Alction categorically axiomatises Actors using the following axioms where $\curvearrowright$ (read as "precedes") is transitive and irreflexive relationship on events and $\mathrm{Info}[\mathrm{x}]$ is the information in the Actor addresses of $\mathrm{x}$ :

- Primitive Actors

$\circ \forall[\mathrm{i}: N]$ i:Actor // natural numbers are Actors

$\circ \forall\left[\mathrm{x}_{1}, \mathrm{x}_{2}\right.$ :Actor $]\left[\mathrm{x}_{1}, \mathrm{x}_{2}\right]$ :Actor // a 2-tuple of Actors is an Actor

- Actor behavior

$\circ \forall[\mathrm{x}:$ Actor, $\mathrm{c}:$ Com $]\left(\nexists\left[\mathrm{c}_{1}:\right.\right.$ Com $\left.] \operatorname{Received}_{\mathrm{x}}\left[\mathrm{c}_{1}\right] \curvearrowright \operatorname{Received}_{\mathrm{x}}[\mathrm{c}]\right) \Rightarrow \mathrm{x}_{\text {received }[\mathrm{c}]}=\mathrm{x}$ initial

$\circ \forall\left[\mathrm{x}:\right.$ Actor, $\mathrm{c}_{1}, \mathrm{c}_{2}:$ Com $]\left(\nexists\left[\mathrm{c}_{3}:\right.\right.$ Com $\left.] \operatorname{After}_{\mathrm{x}}\left[\mathrm{c}_{1}\right] \curvearrowright \operatorname{Received}_{\mathrm{x}}\left[\mathrm{c}_{3}\right] \curvearrowright \operatorname{Received}_{\mathrm{x}}\left[\mathrm{c}_{2}\right]\right)$

$\Rightarrow \mathrm{x}$ received $[\mathrm{c} 2]=\mathrm{x} \cdot \operatorname{after}\left[\mathrm{c}_{1}\right]$

$\circ \forall[\mathrm{x}:$ Actor, c:Com] Finite[Com $\underline{\exists} \mathrm{s} \mapsto \mathrm{s}: \mathrm{x} \cdot \operatorname{sent}[\mathrm{c}]] / /$ only finitely messages are sent while

// processing a communication

- Events

$\circ \forall[\mathrm{c}: C o m] \exists 1[\mathrm{e}:$ Event $] \mathrm{c:Sent} \triangleleft \mathrm{e} \triangleright] \quad / /$ a communication was sent in exactly 1 event

$\circ \forall[\mathrm{c}:$ Com, e1,e2:Event, c:Com] e1:Received [c] $\wedge$ e2:Received $[\mathrm{c}] \Rightarrow \mathrm{e} 1=\mathrm{e} 2$

// a communication is received at most once

$\circ \forall[\mathrm{x}:$ Actor, c:Com $] \mathrm{x}:$ Created $\triangleleft \mathrm{c} \triangleright \Rightarrow$ Initial $_{\mathrm{x}}=$ Creation $[\mathrm{x}]$

// initial event of a created Actor is its creation event 
$\circ \forall[\mathrm{x}:$ Actor, c:Com $]$ Initial $_{\mathrm{x}} \curvearrowright \operatorname{Received}_{\mathrm{x}}[\mathrm{c}] \curvearrowright \operatorname{After}_{\mathrm{x}}[\mathrm{c}]$

$\circ \forall\left[\mathrm{x}:\right.$ Actor, $\mathrm{c}_{1}, \mathrm{c}_{2}$ :Com $] \mathrm{c}_{1} \neq \mathrm{c}_{2} \Rightarrow\left(\operatorname{Received}_{\mathrm{x}}\left[\mathrm{c}_{1}\right] \curvearrowright \operatorname{Received}_{\mathrm{X}}\left[\mathrm{c}_{2}\right] \vee \operatorname{Received}_{\mathrm{x}}\left[\mathrm{c}_{2}\right] \curvearrowright \operatorname{Received}_{\mathrm{X}}\left[\mathrm{c}_{1}\right]\right)$

$/ /$ an Actor imposes an order in which communications are received

$\circ \forall\left[\mathrm{x}:\right.$ Actor, c:Com $\nexists \nexists\left[\mathrm{c}_{1}:\right.$ Com $] \operatorname{Received}_{\mathrm{x}}[\mathrm{c}] \curvearrowright \operatorname{Received}_{\mathrm{x}}\left[\mathrm{c}_{1}\right] \curvearrowright \operatorname{After}_{\mathrm{x}}[\mathrm{c}]$

// no communication is received while an Actor is in its region of mutual exclusion

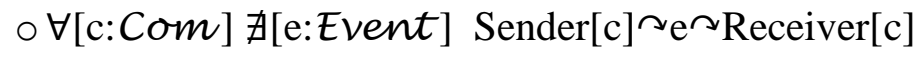

$/ /$ there is no event between sending a receiving a communication

$\circ \forall\left[\mathrm{e}_{1}, \mathrm{e}_{2}, \mathrm{e}_{3}:\right.$ Event $] \mathrm{e}_{1} \curvearrowright \mathrm{e}_{2} \wedge \mathrm{e}_{2} \curvearrowright \mathrm{e}_{3} \Rightarrow \mathrm{e}_{1} \curvearrowright \mathrm{e}_{3} \quad / / \curvearrowright$ is transitive

$\circ$ Event Induction:

$\forall\left[\mathrm{P}\right.$ predicateOn Alcition $_{\text {Event }]}$

( $\forall[\mathrm{x}:$ Actor $] \mathrm{P} \llbracket$ Initial $\mathrm{x} \rrbracket \quad / /$ If $\mathrm{P}$ holds for every initial event of every Actor and

$\Lambda \forall[\mathrm{x}:$ Actor, $\mathrm{c}:$ Com $] \quad / /$ For every Actor and communication

$\mathrm{P} \llbracket$ Received $_{\mathrm{x}}[\mathrm{c}] \rrbracket \quad / /$ If $\mathrm{P}$ holds for a received event of the Actor, then

$\Rightarrow((\forall[\mathrm{e}:$ Activated $\triangleleft \mathrm{c} \triangleright] \mathrm{P} \llbracket \mathrm{e} \rrbracket) \wedge \forall[\mathrm{e}:$ ReceivedNextAfter $\triangleleft \mathrm{c} \triangleright] \mathrm{P} \llbracket \mathrm{e} \rrbracket)$

$/ / \mathrm{P}$ holds for subsequent immediately activated events of the communication

$/ /$ and $\mathrm{P}$ holds for any immediately subsequently received event of the Actor

$\Rightarrow \forall[\mathrm{e}:$ Event $] \mathrm{P} \llbracket \mathrm{e} \rrbracket / /$ then $\mathrm{P}$ holds for every event

- Bits of an address cannot be inferred without being communicated from the creator of the address (cf.

[Hewitt and Baker 1977]):

$\circ \forall\left[\mathrm{e}, \mathrm{e}_{1}\right.$ :Event, c:Com, $\mathrm{x}$ :Actor $] \mathrm{e}_{1}$ :Creattion $\triangleleft \mathrm{c} \triangleright \wedge \mathrm{e}^{\curvearrowright} \operatorname{Received}_{\mathrm{x}}[\mathrm{c}] \Rightarrow \perp=\operatorname{Info}\left[\mathrm{e}_{1}\right] \sqcap \operatorname{Info}[\mathrm{e}]$

// info about the address of a newly created Actor does not provide any

$/ /$ information about addresses in previous events

$\circ \forall\left[\mathrm{e}_{1}, \mathrm{e}_{2}\right.$ :Event, $\mathrm{c}_{1}, \mathrm{c}_{2}$ :Com $] \mathrm{c}_{1} \neq \mathrm{c}_{2} \wedge \mathrm{e}_{1}$ :Creation $\triangleleft \mathrm{c}_{1} \triangleright \wedge \mathrm{e}_{2}$ :Creation $\triangleleft \mathrm{c}_{2} \triangleright \Rightarrow \perp=\operatorname{Info}\left[\mathrm{e}_{1}\right] \sqcap \operatorname{Info}\left[\mathrm{e}_{2}\right]$

$/ /$ info about the address of a newly created Actor does not provide any information

// about address of newly created Actor by a different communication

$\circ \forall\left[\mathrm{e}_{1}, \mathrm{e}_{2}\right.$ :Event, c:Com $] \mathrm{e}_{1} \neq \mathrm{e}_{2} \wedge \mathrm{e}_{1}$ :Creation $\triangleleft \mathrm{c} \triangleright \wedge \mathrm{e}_{2}$ :Creation $\triangleleft \mathrm{c} \triangleright \Rightarrow \perp=\operatorname{Info}\left[\mathrm{e}_{1}\right] \sqcap \operatorname{Info}\left[\mathrm{e}_{2}\right]$

$/ /$ info about the address of a newly created Actor does not provide any information

// about address of any other newly created Actor of the same communication

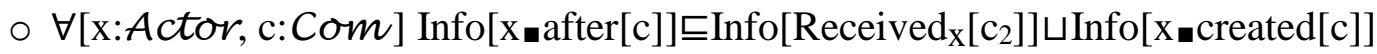

// info about addresses in $\mathrm{x}$ after processing $\mathrm{c}$ is contained in the information when

// $\mathrm{c}$ was received together with info created as a result of processing $\mathrm{c}$

Note that the above axioms do not require that every communication sent must be received. However, ActorScript [Hewitt and Woods assisted by Spurr 2015] provides that every request will either throw a Toolong exception or provide a response which may be a thrown exception from the receiver of the request.

Theorem. Actor Induction, i.e.,

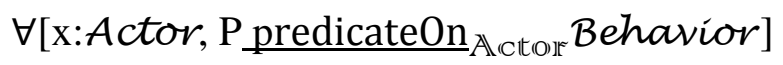

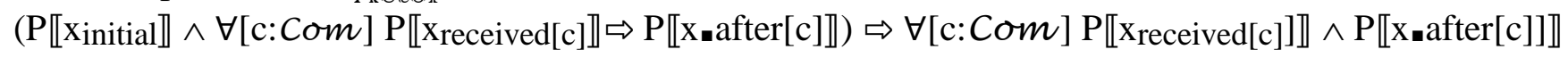

Proof. Follows immediately from Actor Event Induction axiom.

Theorem. $\curvearrowright$ is asymmetric, i.e., $\forall\left[\mathrm{e}_{1}, \mathrm{e}_{2}\right.$ :Event $] \mathrm{e}_{1} \curvearrowright \mathrm{e}_{2} \Rightarrow \neg \mathrm{e}_{2} \curvearrowright \mathrm{e}_{1}$

Theorem. Every event is a receipt or activated by unique communication., i.e.,

$\forall[$ e:Event $] \exists 1[\mathrm{c}: C o m]$ e:Received $\triangleleft \mathrm{c} \triangleright \vee$ e:Activated $\triangleleft \mathrm{c} \triangleright$

Theorem. Digital computation requires time, i.e., $\forall\left[\mathrm{e}_{1}, \mathrm{e}_{2}\right.$ :Event] Finite[Event $\left.\underline{\underline{ }} \mathrm{e} \mapsto \mathrm{e}_{1} \curvearrowright \mathrm{e}^{\curvearrowright} \mathrm{e}_{2}\right]$

Proof. Follows from Actor Event Induction on events that follow e1 in $\curvearrowright$ ordering.

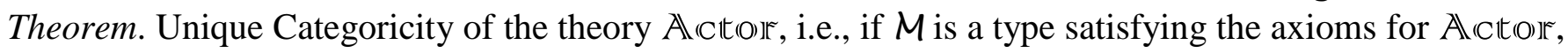
then there is a unique isomorphism between $M$ and TypeI $n \triangleleft \mathbb{A}$ ictione $D$.

Proof. Follows from Actor Event Induction. 
Thesis. Any digital system can be directly modeled and implemented using Actors.

In many practical applications, the parallel $\lambda$-calculus and pure Logic Programs can be thousands of times slower than Actor implementations.

Actor Program Expressions

Eval $\triangleleft t \triangleright:[$ Expression $\triangleleft t \triangleright$ using Environment] $\rightarrow t$ is a procedure [McCarthy et. al. 1962] that corresponds to a universal Turing machine [Turing 1936] as follows:

$\circ$ Eval $\triangleleft$ Expression $\triangleleft t \triangleright \triangleright-[\mathrm{x}] \equiv$ Eval $\triangleleft t \triangleright-[\mathrm{x}$ using EmptyEnvironment $]$

- Eval $\triangleleft$ Identifier $\triangleleft t \triangleright \triangleright-[\mathrm{x}$ using $\mathrm{e}] \equiv \operatorname{Lookup}[\mathrm{x}$ using $\mathrm{e}]$

- Eval $\triangleleft A p p$ lication $\triangleleft t_{1}, t_{2} \triangleright \nabla_{\text {. }}$ [(operator.operand) using $\left.\mathrm{e}\right] \equiv$

(Eval $\triangleleft$ Expression $\triangleleft t_{1} \rightarrow t_{2} \triangleright \triangleright$.[operator using e])

- (Eval $\triangleleft$ Expression $\triangleleft t_{1} \triangleright \triangleright$. [operand using e])

// apply the value of operator to the value of operand

- Eval $\triangleleft$ MappingExpression $\triangleleft t_{1}, t_{2} \triangleright \nabla_{-}[(\mathrm{x} 1 \mapsto$ body $)$ using $] \equiv$

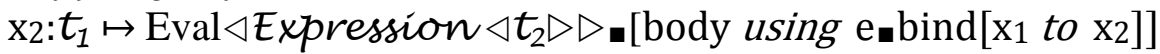

// eval body in a new environment with $\mathrm{x} 1$ bound to $\mathrm{x} 2$ as an extension of $\mathrm{e}$

- Eval $\triangleleft$ Fix Expression $\triangleleft t_{1}, t_{2} \triangleright \triangleright-\left[\right.$ Fix $\triangleleft t_{1}, t_{2} \triangleright$ using $] \equiv$

$$
\mathrm{g}:\left(t_{1} \rightarrow t_{2}\right) \mapsto \underline{\mathrm{Fix}} \triangleleft t_{1}, t_{2} \triangleright . \mathrm{g} \quad / / \underline{\mathrm{Fix}} \triangleleft t_{1}, t_{2} \triangleright . \mathrm{g} \text { is a fixed point of } \mathrm{g}
$$

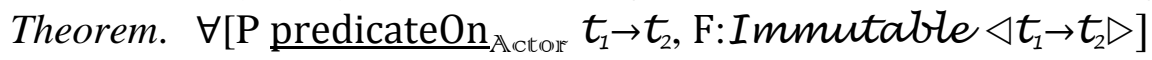

$\left.\left(\mathrm{P} \llbracket \mathrm{F} \cdot\left[\perp \triangleleft t_{1}, t_{2} \triangleright\right] \rrbracket \wedge \forall\left[\mathrm{g}: t_{1} \rightarrow t_{2}\right] \mathrm{P} \llbracket \mathrm{g} \rrbracket \Rightarrow \mathrm{P} \llbracket \mathrm{F} \cdot \mathrm{g}\right] \rrbracket\right) \Rightarrow \mathrm{P} \llbracket \underline{\mathrm{Fix}} \triangleleft t_{1}, t_{2} \triangleright \mathbf{F} \rrbracket$

// where $\perp \triangleleft t_{1}, t_{2} \triangleright \equiv \mathrm{x}: t_{1} \mapsto \perp \triangleleft t_{1}, t_{2} \triangleright \nabla \mathrm{x}$

Proof. Follows immediately from Actor induction axioms applied to the behavior of $\mathrm{F}$.

Indeterminacy is foundational for digital computation

Sequential composition is not foundational because it can be defined as follows:

Eval $\triangleleft$ SequentialExpression $\triangleleft t \triangleright \triangleright .[\{c ; x\}$ using $\mathrm{e}] \equiv$

Eval $\triangleleft$ Expression $\triangleleft t \triangleright \triangleright$. [x using Perform $-[c$ using $\mathrm{e}]]$

// where Perform $-[\mathrm{c}$ using e] is the environment from performing c using e 
On the other hand, non-determinacy can be defined using an implementation Justone that arbitrates as follows:

Chooser implements Choice

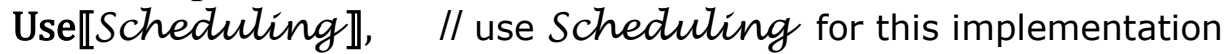

Contained【

// contained locals cannot be sent to other Actors

decided := TheNull Boolean; // the variable decided is initialized as

// the null of type Boolean

pending $\leftarrow$ New Suspended [1]] // where out activity can be suspended

in $1 \mapsto \quad /^{*}$ in 1 message received $/ *\{$

decided $:=\underline{\text { Nullable True; }} \quad$ // decided is assigned to be a nullable of True

Permit pending\} // permit an activity in pending to proceed (if there is one)

in $2 \mapsto / *$ in 2 message received $/ *\{$

decided $:=\underline{\text { Nullable False; }} \quad$ // decided is assigned to be a nullable of False

Permit pending $\} \quad / /$ permit an activity in pending to proceed (if there is one)

out $\mapsto / *$ out message received/* \{

Suspend pending when IsNull decided; // suspend in pending when IsNull[v]

// Require: $\neg$ IsNull[decided]

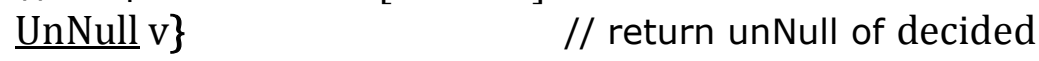

Using Chooser, nondeterministic evaluation of the expression ( $\mathrm{x} 1$ either $\mathrm{x} 2$ ) that either evaluates $\mathrm{x} 2$ or evaluates $\mathrm{x} 2$ can be defined as follows:

Eval $\triangleleft$ EitherExpression $\triangleleft t \triangleright D_{-}[(\mathrm{x} 1$ either $\mathrm{x} 2)$ using $\mathrm{e}] \equiv\{$

anArbiter $\leftarrow$ New Chooser, // identifier anArbiter is bound to a new Chooser

anArbiter.in1, // send in1 to anArbiter

anArbiter.in2, // while concurrently sending in2 to anArbiter

anArbiter.out ?? // test the result when available

True then Eval $\triangleleft$ Expression $\triangleleft t \triangleright D_{-}[\mathrm{x} 1$ using e] $\quad / /$ if True then eval $\mathrm{x} 1$

False then Eval $\triangleleft$ Expression $\triangleleft t \triangleright \nabla_{.}[\mathrm{x} 2$ using $\left.\mathrm{e}]\right\} \quad / /$ if $\underline{\text { False }}$ then eval $\mathrm{x} 1$

Consequently, Church/Turing nondeterministic execution can be defined using Actors, although the indeterminate execution of digital computation cannot in general be implemented using only nondeterministic execution, as shown in this article.

Metatheory of the theory Alction

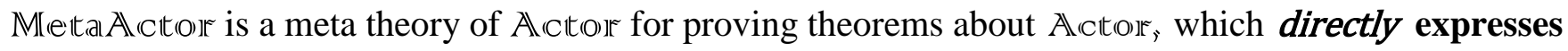
provability of a proposition $\Psi$ in the theory $A_{\text {Action }}$ using $\vdash_{A \text { Acitorf }} \Psi$. (Gödel numbers cannot be used to represent propositions because there are not enough Gödel numbers to represent all uncountably many propositions that are instances of the induction axioms.)

Proof Checkers in the theory Actiour

A proof checker pc:ProofChecker $\triangleleft \mathbb{A}$ cition $\triangleright$ (cf. [Gordon, Milner and Wadsworth 1979]) is a provably total boolean-valued procedure of two arguments that checks if the second argument is validly inferred from the first argument. The following notation (which is part of the theory Alctior) means that pc is proof checker such that proposition $\Psi_{1}$ infers proposition $\Psi_{2}$ in the theory Actiolf (written $\Psi_{1} \vdash \frac{\text { pc }}{\text { Actor }} \Psi_{2}$ ) such

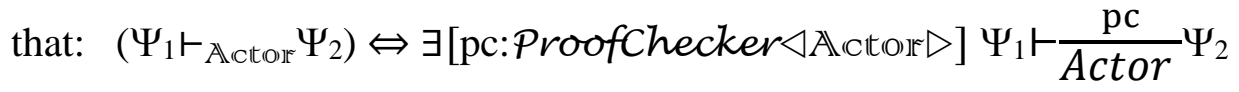


Proof checking in the theory Action is computationally decidable because:

$\forall\left[\right.$ Proposition $\Psi_{1}, \Psi_{2}$; pc:ProofChecker $\triangleleft \mathbb{A}$ cition $\left.\triangleright\right]\left(\Psi_{1} \vdash \frac{\mathrm{pc}}{\text { Actor }} \Psi_{2}\right) \Leftrightarrow \operatorname{pc} n\left[\Psi_{1}, \Psi_{2}\right]=\underline{\text { True }}$ where $\operatorname{pc}_{-}\left[\Psi_{1}, \Psi_{2}\right]$ means the invocation of procedure pc with arguments $\Psi_{1}$ and $\Psi_{2}$. For example, a proof checker for the induction axiom is as follows:

InductionChecker $\left[\Psi_{1}, \Psi_{2}\right] \equiv \Psi_{1}$ ?? $\left(\mathrm{P} \llbracket 0 \rrbracket \wedge \forall[\mathrm{i}: N] \mathrm{P} \llbracket \mathrm{i} \rrbracket \Rightarrow \mathrm{P} \llbracket+{ }_{1}[\mathrm{i}] \rrbracket\right)$ then $\Psi_{2}=\forall[\mathrm{i}: N] \mathrm{P} \llbracket \mathrm{i} \rrbracket$, else $\underline{\text { False }}$ Note that InductionChecker correctly checks uncountably many instances of each of the theory $A$ citions induction axioms.

The rule TheoremUse means that a theorem in Action can be used in proofs in Alction as follows: $\forall\left[\right.$ Proposition $\left.\Psi_{1}, \Psi_{2}\right]$ TheoremUse $\left[\Psi_{1}, \Psi_{2}\right] \equiv \Psi_{2}$ ?? $\left(\vdash_{\text {Alctorr }} \Psi_{1}\right)$ then True else False. Consequently,

$\left(\vdash_{\text {Action }} \Psi\right) \vdash \frac{\text { TheoremUse }}{\text { Actor }} \Psi$. A consequence of TheoremUse is that unrestricted cut-elimination does not hold for the theory Alcition.

There are uncountable proof checkers in the theory $\mathbb{A}$ (ction which is made possible because proof checkers can operate on higher order types, e.g., they are not restricted to strings. For example, there are uncountable proof checkers of the form ForAllEliminationChecker $\triangleleft t \triangleright$ [c] where $t$ is a type and c: $t$ such that ForAllEliminationChecker $\triangleleft t \triangleright[\mathrm{c}]-\left[\Psi_{1}, \Psi_{2}\right] \equiv \Psi_{1} ? ?(\forall[\mathrm{x}: t] \mathrm{P}[\mathrm{x}])$ then $\Psi_{2}=\mathrm{P}[\mathrm{c}]$, else False

Consequently, $(\forall[\mathrm{x}: t] \mathrm{P}[\mathrm{x}]) \vdash \frac{\text { ForAllEliminationChecker } \triangleleft t \triangleright[\mathrm{c}]}{\text { Actor }} \mathrm{P}[\mathrm{c}]$

\section{Types and propositions of the theory Action}

Types and propositions of the theory $A$ citous are axiomatized in terms of each other.

The following axioms hold for TypeIn $\triangleleft \mathbb{A}$ citorr $\triangleright$ (the type of types in the theory $\mathbb{A}$ (ction) because types are intensional:

- $N$ :TypeIn $\triangleleft A \mathbb{A}$ citione $\triangleright / / N$ is type of natural numbers

- $\forall\left[i: N_{+}\right]$Propositionoforder $\triangleleft i \triangleright: T y p e I n \triangleleft A$ icition $\triangleright$

- $\forall\left[t_{1}, t_{2}, t_{3}, t_{4}: T y p e I n \triangleleft A \mathbb{A}\right.$ cition $\left.\triangleright\right]\left[t_{1}, t_{2}\right]=\left[t_{3}, t_{4}\right] \Rightarrow t_{1}=t_{2} \wedge t_{3}=t_{4}$

- $\forall\left[t_{1}, t_{2}, t_{3}, t_{4}:\right.$ TypeIn $\triangleleft \mathbb{A}$ icition $\left.\triangleright\right] t_{1} \rightarrow t_{2}=t_{3} \rightarrow t_{4} \Rightarrow t_{1}=t_{2} \wedge t_{3}=t_{4} \quad / /$ computable procedures

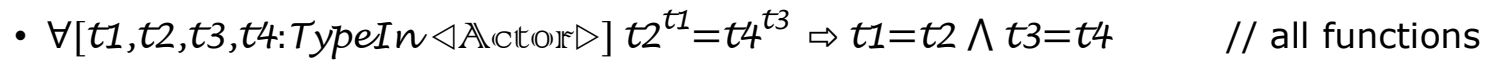

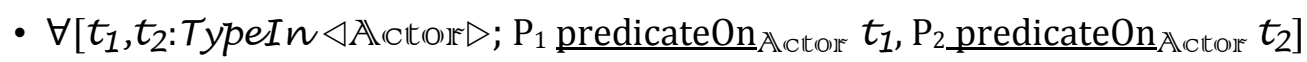

$$
t_{1} \exists \mathrm{P}_{1}=t_{2} \underline{\exists} \mathrm{P}_{2} \Rightarrow t_{1}=t_{2} \wedge \mathrm{P} 1=\mathrm{P}_{2}
$$

For example, $(N \rightarrow N)$ :TypeI $\sim \triangleleft A$ Actiour $\triangleright$, etc.

The following induction axiom holds (cf. [Palmgren 1998, Uemura 2019]), which has uncountable instances.

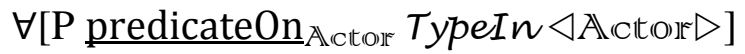

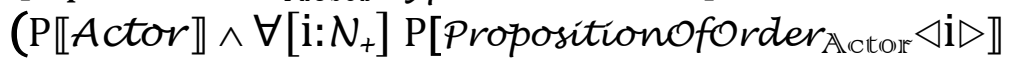

$\wedge \forall\left[t_{1}, t_{2}:\right.$ TypeIn $\triangleleft A$ Action $\left.D\right] \mathrm{P} \llbracket t_{1} \rrbracket \wedge \mathrm{P} \llbracket t_{1} \rrbracket \Rightarrow \mathrm{P} \llbracket\left[t_{1}, t_{2}\right] \rrbracket$

$\wedge \forall\left[t_{1}, t_{2}:\right.$ TypeIn $\triangleleft \mathbb{A}$ iction $\left.\triangleright\right] \mathrm{P} \llbracket t_{1} \rrbracket \wedge \mathrm{P} \llbracket t_{2} \rrbracket \Rightarrow \mathrm{P} \llbracket t_{1} \rightarrow t_{2} \rrbracket$

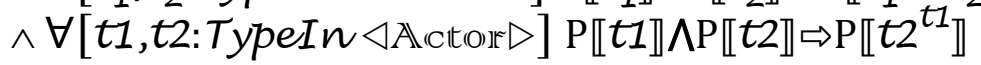

$\wedge \forall[t: T y p e I n \triangleleft A$ iction $\triangleright] \mathrm{P} \llbracket t \rrbracket \mathrm{P} \llbracket T$ ypeot $\triangleleft t \triangleright \rrbracket$

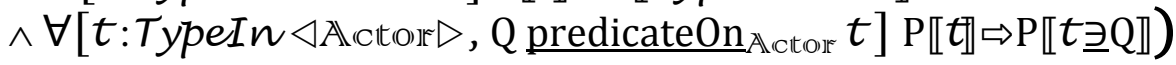

$\Rightarrow \forall[t: T y p e I n \triangleleft A$ action $\triangleright] \mathrm{P} \llbracket t \rrbracket$ 
Theorem Unique categoricity of TypeIn $\triangleleft A \mathbb{A}$ cition $\triangleright$, i.e., if $M$ is a type satisfying the theory $A$ citour,

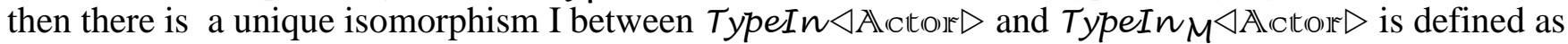
follows:

$\left.\cdot \mathrm{I}\left[t_{1}, t_{2}\right]\right] \equiv\left[\mathrm{I}\left[t_{1}\right], \mathrm{I}\left[t_{2}\right]\right]_{M}$

- $\mathrm{I}\left[t_{1} \rightarrow t_{2}\right] \equiv \mathrm{I}\left[t_{1}\right] \rightarrow \mathrm{I}\left[t_{2}\right]$

- $\mathrm{I}\left[t 2^{t 1}\right] \equiv \mathrm{I}[t 2]^{\mathrm{I}[t 1]}$

- $\mathrm{I}[t \underline{\exists P}] \equiv \mathrm{I}[t] \underline{\exists} M \mathrm{I}[\mathrm{P}]$

The following induction axiom holds for propositions of the theory Alctions (cf. [Martin-Löf 1998, Harrison 2017]), which has uncountable instances:

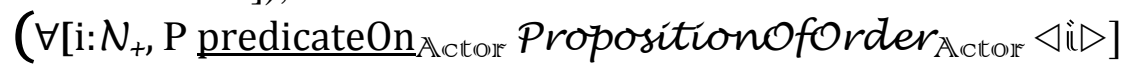

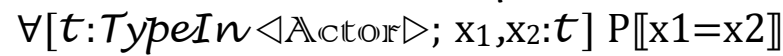

$\wedge \forall\left[t_{1}, t_{2}:\right.$ TypeIn $\triangleleft \mathbb{A}$ cition $\left.\triangleright ; \mathrm{x}: t_{2}\right] \mathrm{P} \llbracket \mathrm{x}: t \rrbracket$

$\wedge \forall\left[\underline{\text { Proposition }}_{\mathbb{A} \text { citour }} \Psi\right] \mathrm{P} \llbracket \Psi \rrbracket \Rightarrow \mathrm{P} \llbracket \neg \Psi \rrbracket$

$\wedge \forall\left[\right.$ Proposition $\left._{\text {Actious }} \Psi_{1}, \Psi_{2}\right] \mathrm{P} \llbracket \Psi_{1} \rrbracket \wedge \mathrm{P} \llbracket \Psi_{2} \rrbracket \Rightarrow \mathrm{P} \llbracket \Psi_{1} \wedge \Psi_{2} \rrbracket$

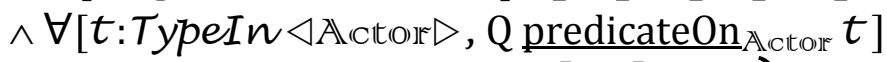

$(\forall[\mathrm{x}: t] \mathrm{P} \llbracket \mathrm{Q} \llbracket \mathrm{x} \rrbracket \rrbracket) \Rightarrow \mathrm{P}[\forall[\mathrm{x}: t] \mathrm{Q} \llbracket \mathrm{x} \rrbracket])$

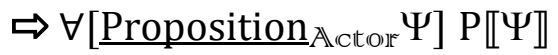

Theorem. Propositions of the theory Alction are characterized up to a unique isomorphism.

Inference in the theory Action

Theorem: Deduction for the theory $A$ ciction, i.e., the following holds:

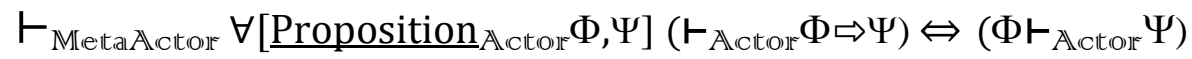

Proof. Suppose $\vdash_{A \text { Actoor }} \Phi \Rightarrow \Psi$ and consequently $\Phi \Rightarrow \Psi$ by TheoremUse. Further suppose $\Phi$. Then $\Psi$ by ChainingForImplication and consequently $\Phi \vdash_{\mathbb{A} \text { citool }} \Psi$ by InferenceIntroduction.

On the other hand suppose $\Phi \vdash_{A \text { Actool }} \Psi$. Further suppose $\Phi$. Then $\Psi$ by ChainingForInference and consequently $\vdash_{\text {Acitorr }} \Phi \Rightarrow \Psi$ by ImplicationIntroduction.

Theorem Inferential Adequacy, i.e., in Mieital Aicition

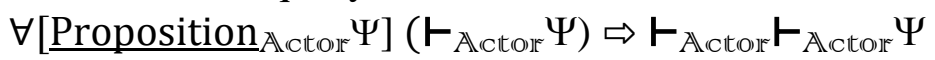

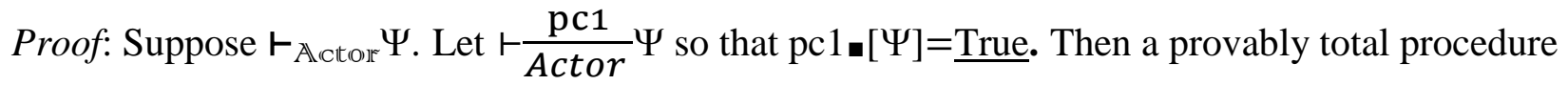

pc2:ProofChecker $\triangleleft \mathbb{A}$ ictions $\triangleright$ can be defined such that pc2 $-\left[\vdash_{A c t i o n} \Psi\right]=$ True. Consequently,

$\vdash_{\text {Alcition }} \boldsymbol{F}_{\text {Alcitorr }} \Psi$.

Theorem: If $M$ is a type satisfying the axioms of the theory $\mathbb{A}$ cition, then there is a unique isomorphism $M$ Model $\triangleleft \mathbb{A}$ icition $D$.

Definition: $\vDash$ can be defined by induction on propositions using the following where $M$ is used for $A$ ctor in Alction $M$ (cf. [Tarski 1936]): $\forall\left[t:\right.$ TypeIn $\left.\triangleleft \mathrm{Action}_{M} \triangleright\right]\left(\vDash_{M} \forall[\mathrm{x}: t] \mathrm{P} \llbracket \mathrm{x} \rrbracket\right) \equiv \forall[\mathrm{x}: t] \vDash_{M} \mathrm{P} \llbracket \mathrm{x} \rrbracket$

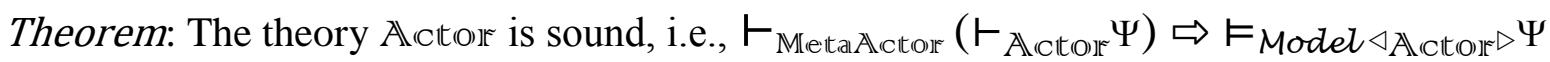

Proof. Follows from induction on types and propositions of Actios:

Theorem. Model $\backslash A$ cition $D$ decides the theory Alction, i.e.,

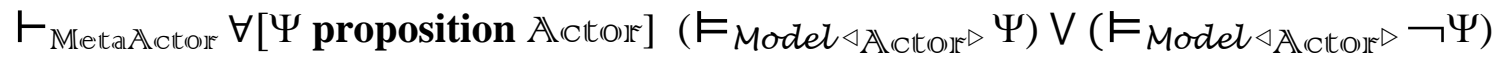

Proof. Immediate from definition of $\vDash$ Model $\triangleleft \mathbb{A}$ iction 
Although Model $\triangleleft \mathbb{A}$ cition $\triangleright$ decides the validity of each proposition of the theory Alction, the theory is computationally and inferentially undecidable

The predicate Halt can be defined as follows on deterministic Boolean expressions:

Halt $[\mathrm{x}$ :Deterministic $\triangleleft$ Boolean $\triangleright] \equiv \exists[\mathrm{y}:$ Boolean $] \mathrm{y}=$ Eval..$[\mathrm{x}]$ Definitions.

- BExpression $\equiv$ Deterministic $\triangleleft$ Expression $\triangleleft$ Boolean $\triangleright \nabla \mid$ String

// deterministic Boolean expression abstracted from a string

- BProcedure $\equiv$ Deterministic $\triangleleft$ BExpression $\lceil$ String $\rightarrow$ Boolean $\triangleright \uparrow$ String

//deterministic Boolean procedure abstracted from a string on an expression abstracted from a string

- Decider $\equiv$ Total $\triangleleft$ BProcedure $\triangleright$

// total BProcedure

Theorem. Halt is computationally undecidable on Boolean expressions abstracted from strings [Church 1935, Turing 1936], i.e., $\nexists[\mathrm{d}$ :Decider $] \forall[\mathrm{x}:$ BExpression $] \mathrm{d} \cdot \mathrm{x}=\underline{\text { True }} \Leftrightarrow \operatorname{Halt}[\mathrm{x}]$

Proof. Suppose to obtain a contradiction that $\mathrm{d}$ :Decider and $\forall[\mathrm{x}:$ BExpression $] \mathrm{d} \cdot \mathrm{x}=$ True $\Leftrightarrow \mathrm{Halt}[\mathrm{x}]$. Let SelfApplier $\equiv$ Deterministic $\triangleleft$ SelfApplier $\rightarrow$ Boolean $\triangleright$ istring so that SelfApplier is a recursively defined type of a deterministic Boolean procedure abstracted from a string. Define the procedure AntiDecider:Selfapplier as follows where ( and ) are used to delimit an expression:

AntiDecider $\equiv \mathrm{p}$ :SelfApplier $\mapsto \mathrm{d} \bullet(\mathrm{p} \bullet \mathrm{p})$ ?? True then LoopForever $\square[]$, False then True Consider the two possibilities for Halt[(AntiDecider-AntiDecider $)]$ to obtain a contradiction as follows:

1. Halt[(AntiDecider $\backsim$ AntiDecider $)]$ :

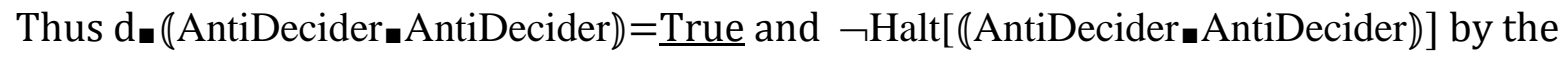
definition of AntiDecider, which is a contradiction.

2. $\neg$ Halt [(AntiDecider-AntiDecider $)]$ :

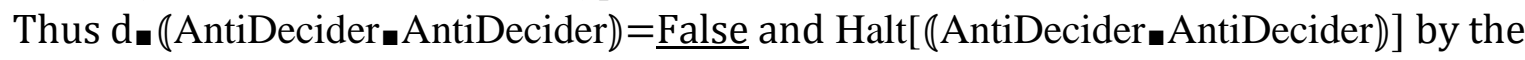
definition of AntiDecider, which is a contradiction.

Consequently, both cases are contradictory and d does not exist.

Theorem. Whether a proposition abstracted from a string is a theorem of Action is computationally undecidable [Church 1935, Turing 1936], i.e., there does not exist a decider d for propositions of the theory

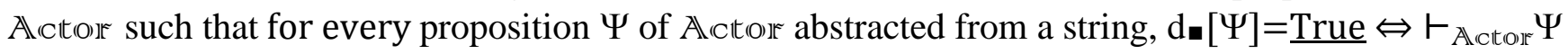

Proof. Follows immediately from the computational undecidability of the halting problem for expressions abstracted from strings because of the following:

$$
\forall[\mathrm{x}: \text { BExpression }] \text { Halt }[\mathrm{x}] \Leftrightarrow \vdash_{\mathbb{A} \text { citions }} \text { Halt }[\mathrm{x}]
$$

Theorem. The theory Action is inferentially undecidable for propositions abstracted from strings, i.e.

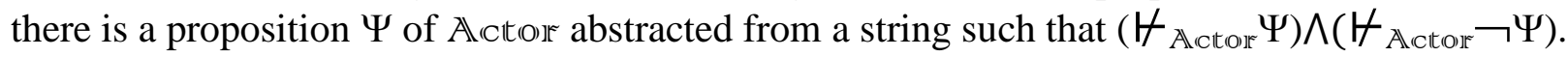

Proof. Suppose to obtain a contradiction that the theory $\mathbb{A}$ citou is inferentially decidable for propositions abstracted from s and consequently $\forall[\mathrm{x}:$ BExpression $]\left(\vdash_{\mathbb{A} \text { citour }} \mathrm{Halt}[\mathrm{x}]\right) \vee\left(\vdash_{\mathbb{A} \text { citor }} \neg \mathrm{Halt}[\mathrm{x}]\right)$

Only countably many instances of the induction axioms could have been used in the proofs because, the halting problem for expressions abstracted from strings is computationally decidable by computationally enumerating the proofs, which is a contradiction.

Theorem. There is a proposition $\Psi$ of $A$ citions $\Gamma$ String such that $\Psi \wedge \forall_{\mathbb{A} \text { citour }} \neg \Psi$.

Proof. By inferential undecidability let $\mathrm{x}$ :BExpression be such that

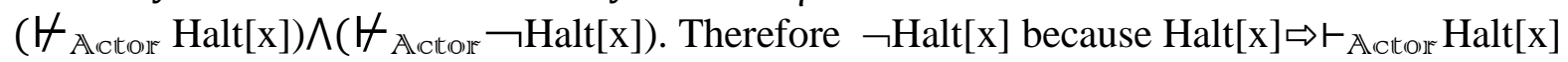

In practice, computational and inferential undecidability of provability, do not impose limitations on the ability to prove theorems for mathematical theories used in Intelligent Systems. Also, computational 
and inferential undecidability of provability of the Actor theory of computation does not necessarily mean that the theory is "incomplete" in the sense that there are useful missing axioms because axioms of the theory characterize Model $\triangleleft \mathbb{A}$ iction $\triangleright \triangleright$ up to a unique isomorphism.

The theory Action is algorithmically inexhaustible

That all the theorems of a theory can be obtained by computationally enumerating them from axioms has long been a default assumption of philosophers of logic. However, the theory Alction violates this assumption because there are uncountable instances of the induction axiom. Uncountability of axiom instances in the theory Alctions raises the following question: What axioms of the theory Action can be expressed in text, i.e., in the theory Alction $\Gamma$ String, i.e., the theory Alction abstracted from strings.

The theory Action $\Gamma$ String has the following induction axiom, which has countable instances because strings are countable:

\section{Definitions.}

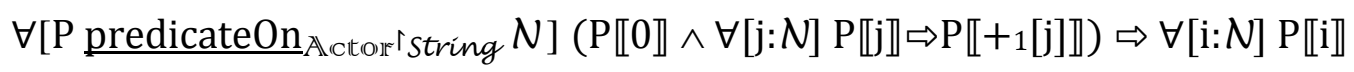

- Total $\triangleleft t \triangleright \equiv($ Deterministic $\triangleleft N \rightarrow t \triangleright \underline{\exists} \mathrm{f} \mapsto \forall[\mathrm{x}: N] \exists[\mathrm{y}: t] \mathrm{f} .[\mathrm{x}]=\mathrm{y})\lceil$ String

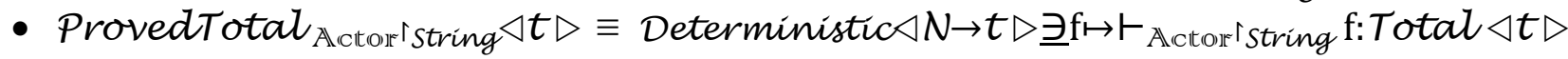

- Onto $\triangleleft t \triangleright \equiv$ Deterministic $\triangleleft N \rightarrow t \triangleright \underline{\exists} \mathrm{f} \mapsto \forall[\mathrm{y}: t] \exists[\mathrm{x}: N] \mathrm{f} \cdot[\mathrm{x}]=\mathrm{y}$

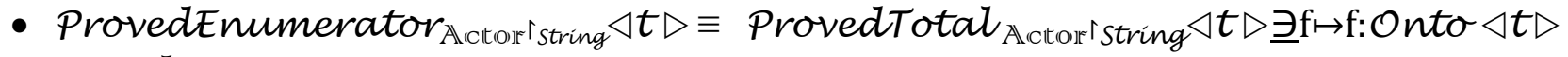

Theorem. Theorem $\triangleleft \mathbb{A}$ cition $\uparrow S t r i n g \triangle$ is computationally enumerable, i.e., there is a procedure

Theorems: ProvedEnumerator Alciour $^{\top}$ String $\triangleleft$ Theorem $\triangleleft \mathbb{A}$ icitio if $\uparrow$ String $\triangleright \triangleright$

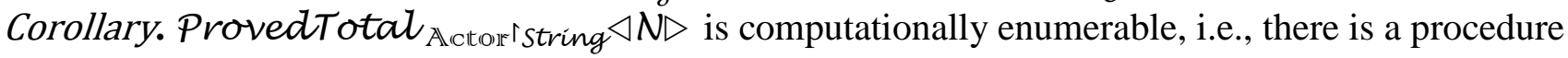

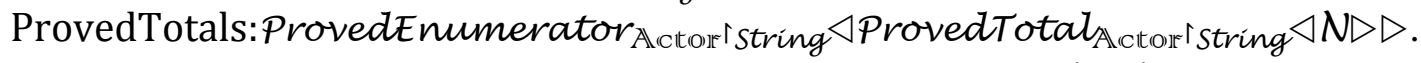

Definition. Define the procedure Diagonal:Deterministic $\triangleleft N \rightarrow N \triangleright \uparrow$ String as follows:

Diagonalø[i:N] $\equiv 1+($ ProvedTotalsø[i] $)$-[i]

Lemma. Diagonal:ProvedTotal Arctore String $_{\text {S }} \backslash N \triangleright$

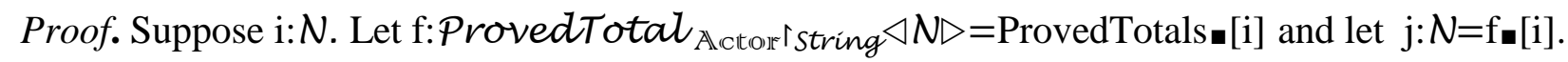

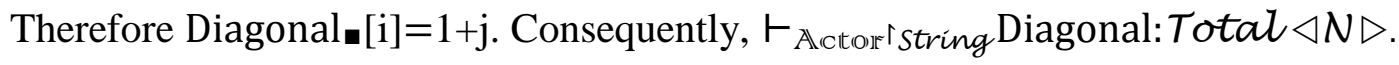

Lemma. $\rightarrow$ Diagonal:ProvedT otal Actour $_{\text {String }} \backslash N D$

Proof. Diagonal differs from every ProvedTotal $\mathbb{A l c t o n e}_{\text {string }} \triangleleft N \triangleright$ enumerated by ProvedTotals.

Theorem. The theory Action $\lceil$ String is inconsistent [Church 1934], i.e.,

$$
\exists\left[\underline{\text { Proposition }}_{\text {Alcitour }} \text { S String } \Psi\right] \vdash_{\text {Accitour }} \text { String } \Psi \wedge \neg \Psi
$$

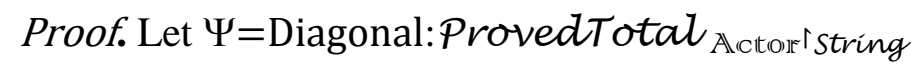

The upshot is that the theory $\mathbb{A}$ citorf is algorithmically inexhaustible, i.e., it is impossible to computationally enumerate theorems of the theory thereby reinforcing the intuition behind [Franzén, 2004]. According to [Church 1934], inconsistency of the theory Actione S String means that "there is no sound basis for supposing that there is such a thing as logic." Contrary to [Church 1934], the conclusion in this article is to abandon the assumption that theorems of a theory must be computationally enumerable while retaining the requirement that proof checking must be computationally decidable. 


\section{MATHEMATICAL THEORIES OF COMPUTER SCIENCE}

Foundational Mathematical Theories of Computer Science

Although theorems of mathematical theories in higher order logic are not computationally enumerable, proof checking is computationally decidable. Strong types can be used categorically axiomatize [Hewitt 20172019] up to a unique isomorphism a mathematical theory $\rceil$ for the model $M$ for each of the following: Natural Numbers, Real Numbers, Ordinals, Computable (Nondeterministic) Procedures, and Actors. Each theory $Т$ has the following properties:

- ТГ is uniquely categorical for Model $\triangle \top \Gamma \triangleright$, i.e., if $X$ satisfies the axioms of $Т$, then is $X$ isomorphic to Model $\triangleleft \top \Gamma \triangleright$, by a unique isomorphism.

- $\Pi$ is sound, i.e., $\left(\vdash_{\rceil} \Psi\right) \Rightarrow \vDash_{\text {Model } \triangleleft\rceil \Gamma \triangleright} \Psi$

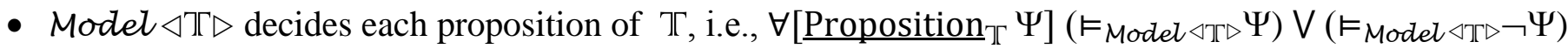

- For all propositions $\Psi$ of $\Pi \Gamma$ and p:ProofChecker $\triangleleft \top \Gamma \triangleright, \vdash \frac{\mathrm{p}}{T} \Psi$ is computationally decidable.

\section{Mathematical Foundations for Computer Science}

Computer Science brought different concerns and a new perspective to mathematical foundations including the following requirements (building on [Maddy 2018]):

- Practicality is providing powerful machinery so that arguments (proofs) can be short and understandable

- Generality is formalizing inference so that all of mathematics can take place side-by-side. Strong types provide generality by formalizing theories of the natural numbers, reals, ordinals, set of elements of a type, groups, lambda calculus, and Actors up to a unique isomorphism side-by-side. For example, the ordinals $\mathcal{O}$ can be axiomatized using strong types so that there is just one model up to a unique isomorphism, which is more general than $1^{\text {st }}$-order set theory because Boolean ${ }^{\mathcal{O}}$ is not part of the cumulative hierarchy of sets.

- Shared Standard of what counts as legitimate mathematics so people can join forces and develop common techniques and technology. According to [Burgess 2015]:

"To guarantee that rigor is not compromised in the process of transferring material from one branch of mathematics to another, it is essential that the starting points of the branches being connected ... be compatible. ... The only obvious way ensure compatibility of the starting points ... is ultimate to derive all branches from a common unified starting point."

This article describes such a common unified starting point including natural numbers, reals, ordinals, set of elements of a type, groups, geometry, algebra, lambda calculus, and Actors that are axiomatized up to a unique isomorphism.

- Abstraction so that fundamental mathematical structures can be characterized up to a unique isomorphism including natural numbers, reals, ordinals, set of elements of a type, groups, lambda calculus, and Actors.

- Guidance is for practioners in their day-to-day work by providing relevant structures and methods free of extraneous factors. This article provides guidance by providing strong parameterized types and intuitive categorical inductive axiomatizations of natural numbers, ordinals, set of elements of a type, lambda calculus, and Actors.

- Meta-Mathematics is the formalization of logic and rules of inference. The mathematical theories described in this article facilitate meta-mathematics because inference is directly on propositions without having to be coded as integers as in [Gödel 1931]. 
- Automation is facilitated in this article by making type checking very easy and intuitive along as well as incorporating Jaśkowski natural deduction for building an inferential system that can be used in everyday work.

- Risk Assessment is the danger of contradictions emerging in classical mathematical theories. This article formalizes long-established and well-tested mathematical practice while blocking all known paradoxes. (See appendix on paradoxes.) Confidence in the consistency of the uniquely categorical theories Alctiont and $\mathrm{O}$ (the theory of the Ordinals) is based on the way that they are inductively constructed bottom-up.

- Monsters [Lakatos 1976] are unwanted elements in models of classical mathematical theories. Alctions precisely characterizes what is digitally computable leaving no room for "monsters" in models. Having a model up to a unique isomorphism in classical mathematical theories is crucial for cybersecurity.

- Inferential completeness is the ability to directly express all inference of classical mathematics. The ordinals $\mathcal{O}$ can be uniquely categorically axiomatized in the theory $(\mathrm{O})$ (using induction for the ordinals in a way analogous to induction on $N$ in the theory $\mathbb{N}$ ) that can directly express proofs of theorems of classical mathematics including [Wiles 1995]. As shown, in this article, additional axioms are needed to axiomatize all digital computation up to a unique isomorphism.

Intuitive categorical inductive axiomatizations of natural numbers, propositions, types, ordinals, set of elements of a type, lambda calculus, and Actors promote confidence in operational consistency.

Consistent mathematical theories can be freely used in (inconsistent) empirical theories without introducing additional inconsistency.

\section{CYBERSECURITY CRISIS}

The current disastrous state of cybersecurity [Sobers 2019, Perlroth, Sanger and Shane 2019] cries out for a paradigm shift.

\section{Nature of Paradigm Shifts}

According to [Kuhn 2012],

"The decision to reject one paradigm is always simultaneously the decision to accept another. First, the new candidate must seem to resolve some outstanding and generally recognized problem that can be met in no other way. Second, the new paradigm must promise to preserve a relatively large part of the concrete problem solving activity that has accrued to science through its predecessor ...

At the start, a new candidate for paradigm shift may have few supporters, and on occasions supporters' motives may be suspect. Nevertheless, if they are competent, they will improve it, explore its possibilities, and show what it would be like to belong to the community guided by it. And as that goes on, if the paradigm is one destined to win its fight, the number and strength of the persuasive arguments in its favor will increase. More scientists will then be converted, the exploration of the new paradigm will go on. Gradually, the number of experiments, instruments, and books upon the paradigm will multiply...

Though a generation is sometimes required to effect the shift, scientific communities have again and again been converted to new paradigms. Furthermore, these conversions occur not despite the fact that scientists are human but because they are. ... Conversions will occur a few at a time until, after the last holdouts have died, the whole profession will again be practicing under a single, but now different paradigm."

\section{Shifting Away from $1^{\text {st }}$-order Logic Foundations}

Computer Science must shift from $1^{\text {st }}$-order logic as the foundation for mathematical theories of Computer Science because of the following deficiencies:

- unwanted monsters in models of theories

- inconsistencies in theories caused by compactness

- being able to infer each and every proposition (including nonsense) from an inconsistency in an empirical theory even though it may not be apparant that the theory is inconsistent. 
Thus Computer Science must move beyond the consensus claimed by [G. H Moore 1988] as follows: "To most mathematical logicians working in the 1980s, first-order logic is the proper and natural framework for mathematics."

The necessity to give up a long-held assumption has often held back the development of science. For example, the Newtonian assumption of absolute space-time had to be given up in the theory of relativity. Also, physical determinacy had to be abandoned in quantum theory. Arthur Erich Has derived the radius of the ground state of the hydrogen atom [Haas 1910], anticipating Niels Bohr work by 3 years. Yet in 1910 Haas's article was rejected and his ideas were termed a "carnival joke" by Viennese physicists. [Hermann 2008] On the other hand, Enrico Fermi received the 1938 Nobel prize for the discovery of the nonexistent elements "Ausonium" and "Hesperium", which were actually mixtures of barium, krypton and other elements. [Fermi 1938]

Identifying and rectifying errors is fundamental to scientific progress. With respect to the subject matter of this article, according to [Church 1934]:

"Indeed, if there is no formalization of logic as a whole [i.e. theorems are not computationally enumerable], then there is no exact description of what logic is, for it in the very nature of an exact description that it implies a formalization. And if there no exact description of logic, then there is no sound basis for supposing that there is such a thing as logic."

Contrary to [Church 1934], the conclusion in this article is to abandon the assumption that theorems of a theory must be computationally enumerable while retaining the requirement that proof checking must be computationally decidable.

\section{Shifting Away from Models of Computation That Are Not Strongly-typed}

Influenced by Turing Machines [Turing 1936], current computer systems are typically not stronglytyped leaving them open to cyberattacks [Hewitt 2019]. Strongly-typed Actors can directly model and implement all digital computation. Consequently, strongly-typed architecture can be extended to microprocessors providing strongly-typed computation all the way to hardware.

How the Computer Science cybersecurity crisis will proceed is indeterminate

Possibilities going forward include the following:

- continue to muddle along without fundamental change

- shift to something along the lines proposed in this article

- shift to some other proposal that has not yet been devised

Cybersecurity issues can provide focus and direction for fundamental research in Computer Science.

\section{RELATED WORK}

Much recent work has centered on constructive type theory (e.g. [Coquand 1986]) which has type $t_{1} \rightarrow_{1} t_{2}$, which is the type of computable procedures on $t_{1}$ into $t_{2}$, but does not have $t_{2}{ }^{t_{1}}$, which is the type of all functions on $t_{1}$ into $t_{2}$. Also, constructive type theory relies on the premise that $\Psi$ is a proposition of theory $\Pi$ if an only if $\Psi$ is a theorem of $\rceil$ with the unfortunate consequence that type checking is computationally undecidable and it is difficult to reason about unprovable propositions.

HOL Light [Harrison 2017] allows more general types than constructive type theory. However, HOL Light is not strongly typed and does not have explicit parameterized types, e.g., a proposition does not have an order, which raises issues with taking fixed points. Also, HOL Light considers two propositions to be equal if they are logically equivalent with the unfortunate consequence that it is difficult to reason about propositions 
that happen to be logically equivalent. For example, all theorems are considered to be equal and can consequently be freely substituted for each other in all terms and propositions.

The Church/Turing model is inadequate for digital computation, as explained in this article. Computing practice diverged from the Church/Turing model when external devices were attached to computers that interacted during computation, which is beyond the algorithmic Church/Turing model. [Hewitt, Bishop, and Steiger 1973] proposed Actors as the universal primitive for digital computation with soon-developed axioms [Greif 1975, Hewitt and Baker 1977, Yonezawa 1977, Hewitt and Atkinson 1979, Atkinson 1980]. [Clinger 1981] and [Agha 1986] developed denotational models but they did not characterize Actors up to a unique isomorphism, as in this article.

[Milner 1993] developed algebraic reduction for use in theories of computation. However even on the same chip, algebraic reduction is not possible for simultaneously sending and receiving a message to an Actor because in general for a message to be received it must go through arbitration with any other messages sent to the Actor. Synchronized message passing can be implemented as follows using a 2-phase commit protocol (cf. [Knabe 1992]) for an implementation I:

synchronizer facet $I$ implements Synchronization $\triangleleft I \triangleright / /$ synchronizer is a facet of $I$

synchronize[aProvider] $\mapsto / /$ request received to synchronize with aProvider ( $\underline{\text { As } I) .(a P r o v i d e r . p r o v i d e) ~ / / ~ p r o c e s s ~ m e s s a g e ~ p r o v i d e d ~ b y ~ a P r o v i d e r ~ i n ~ t h e ~}$

// region of mutual exclusion of this Actor

In this way, the expression f.synchronize[ Actor provide $\mapsto \mathrm{m}]$ for the synchronizer facet $\mathrm{f}$ of an Actor $\mathrm{x}$ processes the message $m$ synchronously. Synchronized messaging requires that the sender must wait to provide a message until it is requested and the recipient must wait for the message to be provided (meanwhile holding up processing of other messages). However, $\mathrm{x}_{\mathbf{m}} \mathrm{m}$ has neither of the extra wait times of synchronized communication nor the requirement that message passing must overlap in time for sender and receiver. Although algebra in the pi-calculus is elegant mathematics, synchronized message passing is not widely used in large software systems because it is slower and less robust than asynchronous message passing.

Also, algebraic refinement orderings [Hoare 2018] can be tricky for programming languages. For example, Interchange[Hoare 2018] does not hold in general for ActorScript [Hewitt and Woods assisted by Spurr 2019], e.g., behavior of the expression $\left\{\mathrm{y} \leftarrow \mathrm{g}_{-}[\mathrm{x}], \mathrm{x} \leftarrow \mathrm{f}_{-}[4]\right.$; $\left.\mathrm{x}+\mathrm{y}\right\}$ (using "," to mean concurrent execution so that g. $[\mathrm{x}]$ and $\mathrm{f}_{\mathbf{2}}$ [4] can be executed concurrently) does not contain the behavior of the expression $\left\{\mathrm{x} \leftarrow \mathrm{f}_{-}[4] ; \mathrm{y} \leftarrow \mathrm{g}_{-}[\mathrm{x}] ; \mathrm{x}+\mathrm{y}\right\}$ (with sequentially executed $\mathrm{x} \leftarrow \mathrm{f}_{-}[4]$ and $\mathrm{y} \leftarrow \mathrm{g}_{-}[\mathrm{x}]$ interchanged from the former expression) because in the latter expression, $\mathrm{x}$ in $\mathrm{g}_{\mathrm{f}}[\mathrm{x}]$ comes from binding the identifier $\mathrm{x}$ in $\mathrm{x} \leftarrow \mathrm{f}_{\mathrm{.}}[4]$ instead of from an outer scope.

The Actor Model attempts to be as general as possible to support direct modeling and efficient implementation of all digital computation. For example, an Actor is not required to have an external mailbox as in Erlang [Armstrong, et. al. 1992]. Requiring an external mailbox is problematical for Actors because the mailbox would itself necessarily be another Actor thereby immediately leading to an infinite regress. Also, requiring the use of external mailboxes can slow message passing between Actors because it would always be necessary to first deposit a message in an Actor's external mailbox so that the message could later be retrieved. Despite some inefficiency and lack of needed functionality (e.g., automatic reclamation of resources of unneeded processes), Erlang has been used to good effect in many impressive projects demonstrating the Actor paradigm.

Requiring use of external mailboxes or requiring use of a synchronous message passing could prevent achieving the goal of less than 10ns average send-to-receipt latency on a chip with thousands of generalpurpose, high-performance cores for the next generation of Intelligent Systems [Hewitt 2019]. 


\section{CONCLUSION}

This article strengthens the position of Computer Science cybersecurity as follows:

- Providing usable theories of standard mathematical theories of computer science (e.g. Natural Numbers and Actors) such that there is only one model up to a unique isomorphism. The approach in this article is to embrace all of the most powerful tools of classical mathematics in order to provide mathematical foundations for Computer Science. Fortunately, these foundations are technically simple so they can be readily automated, which will enable improved collaboration between humans and computer systems.

- Allowing theories to freely reason about theories

- Providing a theory that precisely characterizes all digital computation as well as a strongly-typed programming language that can directly, efficiently, and securely implement every Actor computation.

- Providing in foundation for well-defined classical theories of natural numbers and Actors for use in reasoning by theories of practice in Scalable Intelligent Systems that are (of necessity) pervasively inconsistent.

Blocking known paradoxes makes classical mathematical theories safer for use in Scalable Intelligent Systems by preventing security holes. Consistent strong mathematical theories can be freely used without introducing additional inconsistent information into inferential robust empirical theories that will be the core of future Intelligent Applications.

Inconsistency Robustness [Hewitt and Woods assisted by Spurr 2015] is performance of information systems (including scientific communities) with massive pervasively-inconsistent information. Inconsistency Robustness of the community of professional mathematicians is their performance repeatedly repairing contradictions over the centuries. In the Inconsistency Robustness paradigm, deriving contradictions has been a progressive development and not "game stoppers." Contradictions can be helpful instead of being something to be "swept under the rug" by denying their existence, which has been repeatedly attempted by dogmatic theoreticians (beginning with some Pythagoreans). Such denial has delayed mathematical development.

For reasons of computer security, Computer Science must abandon the thesis that theorems of fundamental mathematical theories must be computationally enumerable. This can be accomplished while preserving almost all previous mathematical work except the $1^{\text {st }}$-Order Thesis [Barwise 1985]. Automation of the proofs in this article is within reach of the state of the art, which will enable better collaboration between humans and computer systems.

Having a powerful system is important because computers must be able to formalize all logical inferences (including inferences about their own inference processes) so that computer systems can better collaborate with humans.

\section{ACKNOWLEDGMENTS}

Extensive conversations with Dan Flickinger, Fanya Montalvo, and Gordon Plotkin and were extremely helpful in developing ideas in this article. Richard Waldinger made very helpful comments. Natarajan Shankar and David Israel pointed out that the article needed to be more explicit on the relationship of Wittgenstein's proof to [Gödel 1931]. Kevin Hammond suggested including the section on related work. Dan Flickinger suggested improvements in the section on paradigm shifts. John Perry provided extensive comments throughout the article. John Woods suggested an improved title. The uniquely categorical theories in this article build on the pioneering work of [Dedekind 1888]. 


\section{APPENDIX: MATHEMATICAL NOTATION}

Notation for mathematical propositions, mathematical terms, and procedural expressions is formalized in this appendix.

Mathematical Proposition is a discrimination of the follow:ng patterns:

$\circ \neg \Psi_{1}, \Psi_{1} \wedge \Psi_{2}$ :Propositionoforder $\triangleleft \mathrm{i} \triangleright$ where $\Psi_{1}, \Psi_{2}:$ Propositionoforder $\triangleleft i \triangleright$ and $\mathrm{i}: N_{+}$

$\circ\left(\mathrm{x}_{1}=\mathrm{x}_{2}\right)$ : Propositionoforder $\triangleleft 1 \triangleright$ where $\mathrm{x}_{1}, \mathrm{x}_{2}$ :Term $\triangleleft t \triangleright$ and $t$ is a type

$\circ$ (x: $t)$ :Propositionoforder $\triangleleft 1 \triangleright$ where $t$ is a type

$\circ \mathrm{P}^{\pi} \mathrm{x} \rrbracket:$ Propositionoforder $\triangleleft \mathrm{i}+1 \triangleright$ where $\mathrm{x}:$ Term $\triangleleft t \triangleright, t$ is a type and $\mathrm{P}:$ Term $\triangleleft$ Proposition $\triangleleft \mathrm{i} \triangleright t \mathrm{t}$ and $\mathrm{i}: N_{+}$

○ $\left(\Psi_{1}+\Psi_{2}\right)$ :Propositionoforder $\triangleleft \mathrm{i} \triangleright$ where $\mathrm{i}: N_{+}$and $\Psi_{1}, \Psi_{2}:$ Propositionoforder $\triangleleft \mathrm{i} \triangleright$

$\circ\left(\Psi_{1} \vdash \frac{\mathrm{p}}{\pi} \Psi_{2}\right):$ Propositionoforder $\triangleleft \mathrm{i} \triangleright$ where $\mathrm{p}:$ Term $\triangleleft$ ProofChecker $\left.\triangleright,\right\rceil$ : Theory, $\Psi_{1}, \Psi_{2}:$ Propositionoforder $\triangleleft i \triangleright$ and $\mathrm{i}: N_{+}$

$\circ\lfloor s\rfloor$ :Propositionoforder $\triangleleft i \triangleright$ is abstraction of s where s:String $\triangle$ Propositionoforder $\triangleleft i \triangleright \triangleright$ with no free variables and i: $N_{+}$

- $\left\lceil\Psi^{\prime}\right\rceil:$ String $\triangleleft$ Propositionoforder $\triangleleft i \triangleright \triangleright$ is quotation of $\Psi$ where $\Psi:$ Propositionoforder $\triangleleft i \triangleright$ iString, and $\mathrm{i}: N_{+}$.

Procedural Expression is a discrimination of the following:

$\circ \mathrm{x}$ : Expression $\triangleleft t \triangleright$ where $\mathrm{x}$ : Constant $\triangleleft t \triangleright$ and $t$ is a type

$\circ \mathrm{x}$ : Expression $\triangleleft t \triangleright$ where $\mathrm{x}$ : Identifier $\triangleleft t \triangleright$ and $t$ is a type

$\circ\left[\mathrm{e}_{1}, \mathrm{e}_{2}\right]$ : Expression $\triangleleft\left[t_{1}, t_{2}\right] \triangleright$ where $\mathrm{e}_{1}$ : Expression $\triangleleft t_{1} \triangleright, \mathrm{e}_{2}$ : Expression $\triangleleft t_{2} \triangleright$, and $t_{1}$ and $t_{2}$ are types

$\circ\left(\mathrm{e}_{1}\right.$ ?? True then $\mathrm{e}_{2}$, False then $\left.\mathrm{e}_{3}\right)$ : Expression $\triangleleft t \triangleright$ where $\mathrm{e}_{1}$ : Expression $\triangleleft$ Boolean $\triangleright$, $\mathrm{e}_{2}, \mathrm{e}_{3}:$ Expression $\triangleleft t \triangleright$ and $t$ is a type

$\circ\left(\mathrm{x}: t_{1} \mapsto \mathrm{y}\right)$ : Expression $\triangleleft t_{1} \rightarrow t_{2} \triangleright$ where $\mathrm{x}$ : Identifier $\triangleleft t_{1} \triangleright, \mathrm{y}$ : Expression $\triangleleft t_{2} \triangleright$ and $t_{1}$ and $t_{2}$ are types

$\circ \mathrm{x} . \mathrm{m}$ : Expression $\triangleleft t_{2} \triangleright$ where $\mathrm{m}$ : Expression $\triangleleft t_{1} \triangleright$, $\mathrm{x}$ is an Actor with a message handler with signature of type Expression $\triangleleft t_{1} \rightarrow t_{2} \triangleright$, and $t_{1}$ and $t_{2}$ are types

$\circ I \llbracket \mathrm{x} 1, \ldots, \mathrm{xn} \rrbracket:$ Expression $\triangleleft I \triangleright$ where $I$ is an Actor implementation and $\mathrm{x} 1, \ldots, \mathrm{xn}$ are expressions.

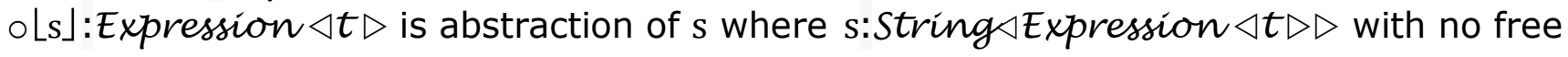
variables and $t$ is a type

$\circ\lceil\mathrm{x}\rceil$ :String $\triangleleft$ Expression $\triangleleft t \triangleright \triangleright$ is quotation of $\mathrm{x}$ where $\mathrm{x}$ : Expression $\triangleleft t \Delta \uparrow S t r i n g$, $\mathrm{i}: N_{+}$and $t$ is a type.

Mathematical Term is a discrimination of the following patterns:

$\circ \mathrm{x}:$ Term $\triangleleft t \triangleright$ where $\mathrm{x}$ :Constant $\triangleleft t \triangleright$ and $t$ is a type

$\circ \mathrm{x}:$ Term $\triangleleft t \triangleright$ where $\mathrm{x}$ Variable $\langle t \triangleright$ and $t$ is a tyne

$\circ\left[\mathrm{x}_{1}, \mathrm{x}_{2}\right]: \operatorname{Term} \triangleleft\left[t_{1}, t_{2}\right] \triangleright$ where $\mathrm{x}_{1}: \operatorname{Term} \triangleleft t_{1} \triangleright, \mathrm{x}_{2}: \operatorname{Term} \triangleleft t_{2} \triangleright$, and $t_{1}$ and $t_{2}$ are types

$\circ\left(\mathrm{x}_{1}\right.$ ?? True then $\left.\mathrm{x}_{2}, \underline{\mathrm{F}} \underline{\underline{c}} \underline{\text { se }} \underline{\text { then }} \mathrm{x}_{3}\right):$ Term $\triangleleft t \downarrow$ where $\mathrm{x}_{1}: \operatorname{Term}<$ Boolean $\triangleright, \mathrm{x}_{2}, \mathrm{x}_{3}:$ Term $\triangleleft t \triangleright$ and $t$ is a type $\left(\left[\mathrm{x}: t_{1}\right] \mapsto \mathrm{y}\right): \operatorname{Term} \triangleleft t_{2}{ }_{t_{1}} \triangleright$ where $\mathrm{x}$ V Variable $\triangleleft t_{1} \triangleright, \mathrm{y}: \operatorname{Term} \triangleleft t_{2} \triangleright$ and $t_{1}$ and $t_{2}$ are typas

of $[\mathrm{x}]: \operatorname{Term} \triangleleft t_{2} \triangleright$ where $\mathrm{f}: \operatorname{Term} \triangleleft t_{2}^{t_{1}} \triangleright, \mathrm{x}: \operatorname{Term} \triangleleft t_{1} \triangleright$, and $t_{1}$ and $t_{2}$ are types 
$\circ\lfloor s\rfloor:$ Term $\triangleleft t \triangleright$ is abstraction of s where s:String $\triangleleft T$ Term $\triangleleft t \triangleright \triangleright$ with no free variables and $t$ is a tipe

$\circ\lceil\mathrm{x}\rceil$ :String $\triangleleft$ Term $\triangleleft t \triangleright \triangleright$ is quotation of $\mathrm{x}$ where $\mathrm{x}$ :Term $\triangleleft t \triangleright \uparrow$ String, $\mathrm{i}: N_{+}$and $t$ is a type.

\section{APPENDIX: MATHEMATICAL PARADOXES}

Inconsistencies in fundamental mathematical theories of Computer Science are dangerous because they can be used to create security vulnerabilities. Strong types are extremely important because they block all known paradoxes including the ones in this appendix.

Burali-Forti/Girad [Burali-Forti 1897, Girard 1972, Coquand 1986, Geuvers 2006]

Although each ordinal $\alpha$ can be strictly embedded as a well-founded order in the ordinals $\mathcal{O}$ and $\alpha={ }_{\mathcal{O}} \mathcal{O} \exists \beta \mapsto \beta: \alpha$ as Ordinals, $\neg \mathcal{O}:(\mathcal{O} \exists \beta \mapsto \beta: \mathcal{O})$ because $\neg \mathcal{O}: \mathcal{O}$, which blocks the paradox. Also, there is no universal type in strongly-typed theories, which blocks [Girard 1972] for the universal type in [Martin-Löf 1971].

Russell [Russell 1902]

o Russell's paradox for sets is resolved as follows: the type of all sets restricted to ones that are not elements of themselves is just the type of all sets because no set is an element of itself.

$\circ$ Russell's paradox for predicates is resolved as follows: The mapping $\mathrm{P} \mapsto \neg \mathrm{P} \llbracket \mathrm{P} \rrbracket$ has no fixed point because $\neg \mathrm{P} \llbracket \mathrm{P} \rrbracket$ has order one greater than the order of $\mathrm{P}$ because $\mathrm{P}$ is a predicate variable.

Berry [Russell 1906]

Berry's Paradox can be formalized using the proposition

Characterize $\triangleleft \mathrm{i} \triangleright \llbracket \mathrm{s}, \mathrm{k} \rrbracket$ meaning that the string s characterizes the integer k as follows where $\mathrm{i}: N_{+}$:

- Berry $\triangleleft i \triangleright \equiv\left(\right.$ Term $\triangleleft$ Propositionoforder $\left.\triangleleft i \triangleright^{N}\right)$ rString

- Characterize $\triangleleft \mathrm{i} \triangleright \llbracket \mathrm{s}:$ Berry $\triangleleft \mathrm{i} \triangleright, \mathrm{k}: N \rrbracket \equiv \forall[\mathrm{x}: N]\lfloor\mathbf{s}\rfloor \llbracket \mathrm{x} \rrbracket \Leftrightarrow \mathrm{x}=\mathrm{k}$

The Berry Paradox is to construct a string for the proposition that holds for integer $n$ if and only if every string with length less than 100 does not characterize $\mathrm{n}$ using the following definition:

BerryString:Berry $\triangleleft i+1 \triangleright \equiv{ }^{c}[\mathrm{j}: N] \mapsto \forall[\mathrm{s}:$ Propositionoforder $\triangleleft i \triangleright \uparrow$ String $]$

Note that

$$
\text { Length }[\mathrm{s}]<100 \Rightarrow \neg \text { Characterize } \triangleleft \mathrm{i} \triangleright \llbracket \mathrm{s}, \mathrm{j} \rrbracket \text { " }
$$

$\circ$ Length[BerryString] $<100$.

$\circ$ Berry $\triangleleft \mathbf{i} \triangleright \exists \underline{s} \mapsto$ Length $[\mathrm{s}]<100$ is finite.

$\circ$ Therefore, BerryNumber is finite where

BerryNumber $\equiv N_{+} \exists \mathrm{j} \mapsto \exists[\mathrm{s}:$ Berry $\triangleleft \mathrm{i} \triangleright]$ Length $[\mathrm{s}]<100 \wedge$ Characterize $\triangleleft \mathrm{i} \triangleright \llbracket \mathrm{s}, \mathrm{j} \rrbracket$

$\circ \exists\left[\mathrm{i}: N_{+}\right]$i:BerryNumber because is $N_{+}$is infinite.

o LeastBerry $\equiv$ Least[BerryNumber]

$\circ\lfloor$ BerryString $\rfloor \llbracket$ LeastBerry $\rrbracket=\forall[\mathrm{s}:$ Berry $\triangleleft \mathbf{i} \triangleright]$ Length $[\mathrm{s}]<100 \Rightarrow \neg$ Characterize $\triangleleft \mathbf{i} \triangleright \llbracket \mathrm{s}$, LeastBerry $\rrbracket$

However BerryString:Berry $\triangleleft i+1 \triangleright$ cannot be substituted for s:Berry $\triangleleft i \triangleright$. Consequently, the Berry

Paradox as follows does not hold:

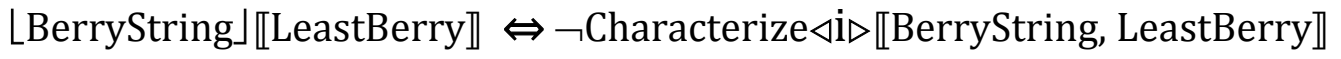

\section{Wittgenstein[Wittgenstein 1978]}

Wittgenstein's Paradox is blocked because the mapping $\Psi \mapsto \not \Psi \Psi$ does not have a fixed point (contra [Gödel 1931]) because the order of $\forall \Psi$ is greater than the order of $\Psi$ since $\Psi$ is a propositional variable. 


\section{Curry [Curry 1941]}

Curry's Paradox is blocked because the mapping $\mathrm{p} \mapsto(\mathrm{p} \Rightarrow \Psi)$ does not have a fixed point because the order of $p \Rightarrow \Psi$ is greater than the order of $p$ since $p$ is a propositional variable.

\section{Löb [Löb 1955]}

Löb's Paradox is blocked because the mapping $\mathrm{p} \mapsto((\vdash \mathrm{p}) \Rightarrow \Psi)$ does not have a fixed point because the order of $(l p) \Rightarrow \Psi$ is greater than the order of $p$ since $p$ is a propositional variable.

Yablo [Yablo 1985]

Yablo's Paradox is blocked because the mapping $\mathrm{P} \mapsto(\forall[\mathrm{i}, \mathrm{j}>\mathrm{i}: N] \neg \mathrm{P} \llbracket \mathrm{j} \rrbracket)$ does not have a fixed point because the order of $\forall[\mathrm{i}, \mathrm{j}>\mathrm{i}: N] \neg \mathrm{P} \llbracket \mathrm{j} \rrbracket$ is one great than the order of $\mathrm{P}$ since $\mathrm{P}$ is a predicate variable [cf. Priest 1997].

\section{APPENDIX: ORDINALS AND NATURAL NUMBERS}

\section{Theory of Natural Numbers}

The mathematical theory $\mathbb{N}$ that axiomatises the Natural Numbers $N$ has the following axioms building on [Dedekind 1888]:

- $0: N$

$+{ }_{1}: N^{N}$

// 0 is of type $\mathrm{N}$

- $\nexists[\mathrm{i}: N]+1[\mathrm{i}]=0$

$/ /+1$ (successor) is of type $N^{N}$

- $\forall[\mathrm{i}, \mathrm{j}: N]+1[\mathrm{i}]=+1[\mathrm{j}] \Rightarrow \mathrm{i}=\mathrm{j}$

$/ / 0$ is not a successor

$/ /+_{1}$ is 1 to 1

In addition, the theory $\mathbb{N}$ has the following induction axiom, which has uncountable instances:

$$
\forall[\mathrm{P} \text { predicateOn } N](\mathrm{P} \llbracket 0 \rrbracket \wedge \forall[\mathrm{j}: N] \mathrm{P} \llbracket \mathrm{j} \rrbracket \Rightarrow \mathrm{P} \llbracket+1[\mathrm{j}] \rrbracket) \Rightarrow \forall[\mathrm{i}: N] \mathrm{P} \llbracket \mathrm{i} \rrbracket
$$

Theorem [cf. Dedekind 1888]: If $M$ be a type satisfying the axioms of the theory $\mathbb{N}$, then there is a unique isomorphism I: $M^{\text {Modek } \triangle \mathbb{N} \triangleright}$ defined as follows:

- Define by induction on TypeIn $\triangleleft \mathbb{N} \triangleright]$

$$
\begin{aligned}
& \circ \mathrm{I}[N] \equiv M \\
& \circ \mathrm{I}\left[\left[t_{1}, t_{2}\right]\right] \equiv\left[\mathrm{I}\left[t_{1}\right], \mathrm{I}\left[t_{2}\right]\right]_{M} \\
& \circ \mathrm{I}\left[t 2^{t 1}\right] \equiv \mathrm{I}[t 2] \\
& \circ \forall[\mathrm{P} \text { predicateOn } \\
& \mathbb{N} \text { TypeIn } \triangleleft \mathbb{N} \triangleright] \mathrm{I}[t \underline{\exists} \mathrm{P}] \equiv \mathrm{I}[t] \underline{\exists}[\mathrm{P}]
\end{aligned}
$$

- Define by induction on TypeIn $\triangleleft \mathbb{N} \triangleright]$

$\circ$ Define by induction on $N$

- $\mathrm{I}[0] \equiv 0_{M}$

- $\mathrm{I}\left[+_{1}[\mathrm{j}]\right] \equiv+_{1}^{M}[\mathrm{I}[\mathrm{j}]]$

$\circ$ if $x:\left[t_{1}, t_{2}\right]$, then $I[x] \equiv\left[I\left[1^{\text {st }}[\mathrm{x}]\right], \mathrm{I}\left[2^{\mathrm{nd}}[\mathrm{x}]\right]\right]_{M}$

$\circ$ if $\mathrm{x}: t 2^{t 1}$, then $\mathrm{I}[\mathrm{x}] \equiv \mathrm{y}: \mathrm{I}\left[t_{2}\right] \mapsto \mathrm{I}\left[\mathrm{x}\left[\mathrm{I}^{-1}[\mathrm{y}]\right]\right] \quad$ // inductive hypothesis for I on $t 2$

$\mathrm{I}$ is a unique isomorphism because of the following;

- I is defined on TypeIn $\triangleleft \mathbb{N} \triangleright$

- $\mathrm{I}$ is $1-1$

- I is onto $M$

- I is a homomorphism

- $\mathrm{I}^{-1}$ is a homomorphism

- If $\mathrm{g}$ is an isomorphism of Model $\triangleleft \mathbb{N} \triangleright$ with $M$, then $g=I$ 
Corollary There are no infinite numbers (monsters) in models of the theory $\mathbb{N}$, e.g., if $M$ satisfies the axioms of the theory $\mathbb{N}$ for $N$, then $\nexists[\mathrm{j}: M] \forall[\mathrm{i}: N] \mathrm{i}<\mathrm{j}$

\section{Theory of Ordinals}

The theory $(\mathrm{O})$ that axiomatises the ordinals $\mathcal{O}$ has the following axioms in addition to the axioms for the theory $\mathbb{N}$ (where $\forall[\alpha, \beta: \mathcal{O}] \alpha: \beta \Leftrightarrow \alpha<\beta)$ :

- $0: \mathcal{O}$

- $\nexists[\alpha: \mathcal{O}] \alpha<0$

- $+1: 1 T \sigma 1 \triangleleft \mathcal{O}, \mathcal{O} \triangleright$

- $\forall[\alpha, \beta, \gamma: \mathcal{O}] \alpha<\beta \wedge \beta<\gamma \Rightarrow \alpha<\gamma$

- $\forall[\alpha, \beta,: \mathcal{O}] \alpha<\beta \vee \alpha=\beta \vee \alpha>\beta$

- $\forall\left[\mathrm{P}\right.$ predicateOn $\left.\mathrm{n}_{\mathrm{O}}, \mathcal{O}\right]$ Least $[\mathrm{P}]: \mathcal{O}$ axiomatized by cases as follows:

1. $\nexists[\alpha: \mathcal{O}] \mathrm{P} \llbracket \alpha \rrbracket:$ Least $[\mathrm{P}] \equiv 0$

2. $\exists[\alpha: \mathcal{O}] \mathrm{P} \llbracket \alpha \rrbracket: \mathrm{P} \llbracket$ Least $[\mathrm{P}] \rrbracket \wedge \forall[\alpha: \mathcal{O}] \mathrm{P} \llbracket \alpha \rrbracket \Rightarrow$ Least $[\mathrm{P}] \leqq \alpha$

- $\forall[\alpha: \mathcal{O}] \omega_{\alpha}: \mathcal{O}$ axiomatized by ordinal induction as follows:

3. $\omega_{0} \equiv N$

4. $\omega_{\alpha+1} \equiv \operatorname{Least}\left[\beta \mapsto 1\right.$ To1 $\left[\right.$ Boolean $\left.\left.\omega_{\alpha \triangleright}\right]\right] \wedge 1$ To1 $\left[\omega_{\alpha+1}\right.$, Boolean $\left.\omega_{\alpha}\right]$

5. $\omega_{\alpha} \equiv$ Least $\left[\beta \mapsto \forall[\gamma<\alpha] \beta>\omega_{\gamma}\right] \wedge \forall[\gamma<\alpha] \omega_{\alpha}>\omega_{\gamma}$

In addition, the theory $(O)$ has the following induction axiom, which has uncountable instances:

$\forall[\mathrm{P}$ predicateOn $(\mathrm{O}) \mathcal{O}](\mathrm{P} \llbracket 0 \rrbracket \wedge \forall[\alpha: \mathcal{O}] \forall[\beta<\alpha: \mathcal{O}] \mathrm{P} \llbracket \beta \rrbracket \Rightarrow \mathrm{P} \llbracket \alpha] \rrbracket) \Rightarrow \forall[\alpha: \mathcal{O}] \mathrm{P} \llbracket \alpha \rrbracket$

Theorem: $\mathcal{O}$ is well-ordered by <, i.e., $\nexists\left[\mathrm{f}: \mathcal{O}^{N}\right] \forall[\mathrm{i}: N] \mathrm{f}[\mathrm{i}+1]<\mathrm{f}[\mathrm{i}]$

Theorem: If $M$ is a type satisfying the axioms of the theory $(\mathrm{O})$, then there is a unique isomorphism I: Model $\triangleleft(O) \triangleright$ defined as follows:

- Define by induction on TypeI $\sim \triangleleft(O) \triangleright$

$\circ \mathrm{I}[\mathcal{O}] \equiv M$

$\circ \mathrm{I}\left[\left[t_{1}, t_{2}\right]\right] \equiv\left[\mathrm{I}\left[t_{1}\right], \mathrm{I}\left[t_{2}\right]\right]_{M}$

$\circ \mathrm{I}\left[t 2^{t 1}\right] \equiv \mathrm{I}[t 2]^{\mathrm{I}[t 1]}$

$\circ \forall[\mathrm{P}$ predicateOn $(\mathrm{O})$ TypeI $\sim \triangleleft(\mathrm{O}) \triangleright, t:$ TypeI $\sim \triangleleft(\mathrm{O}) \triangleright] \mathrm{I}[t \underline{\exists} \mathrm{P}] \equiv \mathrm{I}[t] \underline{\mathrm{I}}[\mathrm{P}]$

- Define by induction on TypeI $\sim \triangleleft(O) \triangleright$

$\circ$ Define by ordinal induction on $\mathcal{O}$

- $\mathrm{I}[0] \equiv 0_{M}$

- $\mathrm{I}[\alpha+1] \equiv \mathrm{I}[\alpha]+M^{1}$

- I $[\alpha] \equiv \operatorname{Least}_{M}[\beta \mapsto \beta \geqq M I[\alpha]]$

$\circ$ if $\mathrm{x}:\left[t_{1}, t_{2}\right]$, then $\mathrm{I}[\mathrm{x}] \equiv\left[\mathrm{I}\left[1^{\mathrm{st}}[\mathrm{x}]\right], \mathrm{I}\left[2^{\mathrm{nd}}[\mathrm{x}]\right]\right]_{M}$

$\circ$ if $\mathrm{x}: t 2^{t 1}$, then $\mathrm{I}[\mathrm{x}] \equiv \mathrm{y}: \mathrm{I}\left[t_{2}\right] \mapsto \mathrm{I}\left[\mathrm{x}\left[\mathrm{I}^{-1}[\mathrm{y}]\right]\right] \quad / /$ inductive hypothesis for $\mathrm{I}$ on $t_{2}$

TypeI $\sim \triangleleft(\mathrm{O}) \triangleright$ is a strict generalization of sets in $1^{\text {st }}$-order set theory, e.g., Boolean ${ }^{\mathcal{O}}$ is not in the cumulative hierarchy of sets. 


\section{REFERENCES}

G. Agha. Actors: A Model of Concurrent Computation in Distributed Systems MIT EECS Doctoral Dissertation. 1986.

M. Amborn. Facet-Oriented Program Design. LiTH-IDA-EX-04/047-SE Linkőpings Universitet. 2004.

J. Armstrong, B. Däcker, S. Virding, and M. Williams. Implementing a functional language for highly parallel real-time applications Software Engineering for Telecommunication Switching Systems. Ericson. April 1992.

S. Artemov. The Provability of Consistency ArXiv. March 18, 2019.

R. Atkinson. Automatic Verification of Serializers MIT Doctoral Dissertation. June, 1980.

J. Avigad, G. Ebner, and S. Ullrich. The Lean Reference Manual: Release 3.3.0. September 6. 2018.

S. Awodey and E. Reck. Completeness and Categoricity. Parts I and II: Nineteenth-century Axiomatics to Twentieth-century Metalogic. History and Philosophy of Logic. Vol. 23. 2002.

J. Barwise. Model-Theoretic Logics: Background and Aims Model Theoretic Logics. Springer-Verlag. 1985.

C. Benzmüller, N. Sultana, L. Paulson and F. Theiß. The Higher-Order Prover Leo-II Journal of Automated Reasoning. Vol. 55. Issue 4. December 2015.

N. Bourbaki. Elements of Mathematics Springer. 1939-2016.

P. Brinch Hansen. Monitors and Concurrent Pascal: A Personal History SIGPLAN Notices. March 1993.

C. Burali-Forti. Una questione sui numeri transfiniti Rendiconti del Circolo Matematico di Palermo. 1897.

J. Burgess. Rigor and Structure Oxford University Press. 2015.

A. Church. A set of postulates for the foundation of logic Annals of Mathematics. Series 2. 33 (2). 1932.

A. Church. The Richard Paradox. Proceedings of American Mathematical Society. Vol. 41. No. 6. 1934.

W. Clinger. Foundations of Actor Semantics MIT Mathematics Doctoral Dissertation. June 1981.

T. Coquand. An Analysis of Girard's Paradox INRIA. Report 531. May 1986.

T. Coquand and G. Huet. The calculus of constructions. Technical Report 530, INRIA, Centre de Rocquencourt, 1986.

E. Crahen. Facet: A pattern for dynamic interfaces. CSE Dept. SUNY at Buffalo. July 22, 2002.

H. Curry. Some Aspects of the Problem of Mathematical Rigor Bulletin of the American Mathematical Society Vol. 4. 1941.

O. Dahl and K. Nygaard. Class and subclass declarations IFIP TC2 Conference on Simulation Programming Languages. May 1967.

R. Dedekind. What are and what should the numbers be? Friedr. Vieweg \& Sohn, 1888. Translated by David E. Joyce, Clark University, Dec. 2005; https://mathcs.clarku.edu/ djoyce/numbers/dedekind.pdf

E. Fermi. Artificial radioactivity produced by neutron bombardment Nobel Lecture. December 12, 1938.

J. Girard. Interprétation fonctionnelle et Élimination des coupure de l'arithmétique d'ordre supérieur These d'Etat. Paris VII. 1972.

H. Geuvers (In)consistency of Extensions of Higher Order Logic and Type Theory Types. April 18-21, 2006.

K. Gödel. The completeness of the axioms of the functional calculus of logic Monatshefte für Mathematik und Physik 3. 1930

K. Gödel. On formally undecidable propositions of Principia Mathematica Monatshefte für Mathematik und Physik. 1931. Translation in From Frege to Gödel: A Source Book in Mathematical Logic. Harvard University Press.

M. Gordon, R. Milner and C. Wadsworth. (1979) Edinburgh LCF: A Mechanised Logic of Computation Lecture Notes in Computer Science. Vol. 78. Springer-Verlag. 1979.

I. Greif. Semantics of Communicating Parallel Processes MIT EECS Doctoral Dissertation. August 1975. 
A. E. Haas. Uber die elektrodynamische Bedeutung des Planckschen Strahlungsgesetzes und uber eine neue Bestimmung des elektrischen Elementarquantums und der dimension des wasserstoffatoms. Sitzungsberichte der kaiserlichen Akademie der Wissenschaften in Wien. 1910.

J. Harrison. HOL Light Tutorial Intel Corporation. January 14, 2017.

C. Hewitt. Planner: A Language for Proving Theorems in Robots IJCAI. 1969.

C. Hewitt, P. Bishop, and R. Steiger. A Universal Modular Actor Formalism for Artificial Intelligence IJCAI. 1973.

C. Hewitt and H. Baker. Laws for Communicating Parallel Processes IFIP-77, August 1977

C. Hewitt and R. Atkinson. Specification and Proof Techniques for Serializers IEEE Journal on Software Engineering. January 1979.

C. Hewitt. Strong Types for Direct Logic. HAL Archive; 2017-2019. https://hal.archives-ouvertes.fr/hal$\underline{01566393}$

C. Hewitt. Citadels: Faster Response Time and Better Information Integration Than Datacenters of Competing Companies Social Science Research Network. Working Paper 2836282. 2016-2019.

https://papers.ssrn.com/sol3/papers.cfm?abstract_id=2836282

C. Hewitt. Building and Deploying Scalable Intelligent Systems by 2025 Video of Stanford University EE380 Colloquium. January 23, 2019.

http://web.stanford.edu/ class/ee380/Abstracts/190123.html

C. Hewitt and J. Woods assisted by Jane Spurr. Inference Robustness Studies in Logic. In press 2019.

A. Hermann. Arthur Erich Haas The Columbia Encyclopedia, 6th ed. 2008.

T. Hoare Monitors: An Operating System Structuring Concept CACM. October 1974.

T. Hoare Algebra, Logic, Geometry: at the Foundations of CS ICFEM. November 14, 2018.

ISO. Programming languages -- C++ ISO/IEC 14882:2017. December 2017.

K. Kahn. A Computational Theory of Animation MIT EECS Doctoral Dissertation. August 1979.

R. Karp and R. Miller. Parallel program schemata: A mathematical model for parallel computation Switching and Automata Theory. October 1967.

F. Knabe A Distributed Protocol for Channel-Based Communication with Choice PARLE'92.

W. Kornfeld. Parallelism in Problem Solving MIT EECS Doctoral Dissertation. August 1981.

T. Kuhn. The Structure of Scientific Revolutions. 50th anniversary edition University of Chicago Press. 2012.

I. Lakatos. Proofs and Refutations. Cambridge University Press. 1976.

M. Löb. Solution of a problem of Leon Henkin Journal of Symbolic Logic. Vol. 20. 1955.

P. Maddy. What do we want a foundation to do? Comparing set-theoretic, category-theoretic, and univalent approaches Reflections on Foundations: Univalent Foundations, Set Theory and General Thoughts. 2018.

P. Martin-Löf. A Theory of Types Stockholm University. Technical Report 71-3. 1971.

P. Martin-Löf. An intuitionistic theory of types in Twenty-Five Years of Constructive Type Theory Oxford University Press. 1998.

J. McCarthy, P. Abrahams, D. Edwards, T. Hart, and M. Levin, LISP 1.5 Programmer's Manual 1962.

R. Milner. Elements of interaction: Turing award lecture CACM. January 1993.

G. H. Moore. The Emergence of First-Order Logic History and

Philosophy of Modern Mathematics. Minnesota Studies in the Philosophy of Science. Volume XI. 1988.

E. Palmgren. On Universes in Type Theory Twenty Five Years of Constructive Type Theory. Oxford University Press. 1998.

G. Plotkin. A powerdomain construction SIAM Journal of Computing. September 1976.

N. Perlroth, D. Sanger and S. Shane. How Chinese Spies Got the N.S.A. 's Hacking Tools, and Used Them for Attacks. New York Times. May 6, 2019.

G. Priest. Yablo's Paradox Analysis 57. 1997. 
B. Russell. Les paradoxes de la logique Revue de métaphysique et de morale. 1906.

B. Russell. Mathematical Logic as Based on the Theory of Types American Journal of Mathematics. 30 (3). 1908.

B. Russell. Logical positivism Revue internationale de philosophie. Vol. 4. 1950.

S. G. Shanker. Wittgenstein's Remarks of the Significance of Gödel's Theorem Godel's Theorem in Focus. Croom Helm. 1988.

R. Sobers. 60 Must-Know Cybersecurity Statistics for 2019. Varonis. April 17, 2019.

A. Tarski. Der Wahrheitsbegriff in den formalisierten Sprachen Studia Philosophica. 1. 1936

A. Turing. On Computable Numbers, with an Application to the Entscheidungsproblem Proceedings of the London Mathematical Society. 2. 42. 1936.

T. Uemura. A General Framework for the Semantics of Type Theory Types. June 11-14, 2019.

A. Urquhart. Russell and Gödel Bulletin of Symbolic Logic. Volume 22, Number 4, December 2016.

L. Wittgenstein. Remarks on the Foundations of Mathematics, Revised Edition Basil Blackwell. 1978.

J. Woods. Errors of Reasoning. Naturalizing the Logic of Inference Studies in Logic. 2013.

J. Woods. How paradox fares in Inconsistency Robust Logic and beyond: Computational and naturalized approaches Inference Robustness, Studies in Logic. 2019.

S. Yablo. Truth and reflection Journal of Philosophical Logic. 14 (2). 1985.

A. Yonezawa Specification and Verification Techniques for Parallel Programs Based on Message Passing Semantics MIT EECS Doctoral Dissertation. December 1977. 